\title{
Comparative biological evaluation and G-quadruplex interaction studies of two new families of organometallic gold(I) complexes featuring N-heterocyclic carbene and alkynyl ligands
}

Samuel M. Meier-Menches, ${ }^{\mathrm{a},+}$ Brech Aikman, ${ }^{\mathrm{a}+}$ Daniel Döllerer, ${ }^{\mathrm{a}}$ Wim T. Klooster, ${ }^{\mathrm{b}}$ Simon J. Coles, ${ }^{\mathrm{b}}$ Nicolò Santi, ${ }^{\mathrm{a}}$ Louis Luk, ${ }^{\mathrm{a}}$ Angela Casini,${ }^{\mathrm{a}, \mathrm{c} *}$ and Riccardo Bonsignore ${ }^{\mathrm{a}, *}$

${ }^{a}$ School of Chemistry, Cardiff University, Main Building, Park Place, Cardiff CF103AT, UK.

${ }^{\mathrm{b}}$ School of Chemistry, University of Southampton, Southampton SO17 1BJ, United Kingdom.

${ }^{c}$ Department of Chemistry, Technical University of Munich, Lichtenbergstr. 4, 85748 Garching, Germany.

${ }^{+}$shared first authors

*Corresponding authors: E-mail: casinia@ cardiff.ac.uk; bonsignorer@ cardiff.ac.uk; Ph/Fax: +44 (0)29 20876364

Keywords: gold(I) organometallics; N-heterocyclic carbenes; alkynyl ligands; G-quadruplexes; cancer.

\section{Abstract}


Experimental organometallic gold(I) compounds hold promise for anticancer therapy. This study reports the synthesis of two novel families of gold(I) complexes, including N1-substituted bis-Nheterocyclic carbene $(\mathrm{NHCs})$ complexes of general formula $\left[\mathrm{Au}(\mathrm{N} 1-\mathrm{TBM})_{2}\right] \mathrm{BF} 4(\mathrm{~N} 1-\mathrm{TBM}=\mathrm{N} 1$ substituted 9-methyltheobromin-8-ylidene) and mixed gold(I) NHC-alkynyl complexes, [Au(N1TBM)alkynyl]. The compounds were fully characterized for their structure and stability in aqueous environment and in the presence of $\mathrm{N}$-acetyl cysteine by nuclear magnetic resonance (NMR) spectroscopy. The structures of bis(1-ethyl-3,7,9-trimethylxanthin-8-ylidene)gold(I), (4ethynylpyridine)(1,9-dimethyltheobromine-8-ylidene)gold(I) and of (2,8-Diethyl-10-(4ethynylphenyl)-5,5-difluoro-1,3,7,9-tetramethyl-5H-4 $\lambda^{4}, 5 \lambda^{4}$-dipyrrolo[1,2-c:2',1'-

$f][1,3,2]$ diazaborinine $)(1,3,7,9$-tetramethylxanthin-8-ylidene)gold(I) were also confirmed by X-ray diffraction analysis. The compounds were studied for their properties as DNA G-quadruplexes (G4s) stabilisers by fluorescence resonance energy transfer (FRET) DNA melting. Only the cationic $\left[\mathrm{Au}(\mathrm{N} 1-\mathrm{TBM})_{2}\right] \mathrm{BF}_{4}$ family showed moderate $\mathrm{G} 4$ stabilization properties with respect to the previously reported benchmark compound $\left[\mathrm{Au}(9 \text {-methylcaffein-8-ylidene })_{2}\right]^{+}(\mathbf{A u T M X} \mathbf{T})$. However, the compounds also showed marked selectivity for binding to G4 structures with respect to duplex DNA in competition experiments. For selected complexes, the interactions with G4s were also confirmed by circular dichroism (CD) studies. Furthermore, the gold(I) complexes were assessed for their antiproliferative effects in human cancer cells in vitro, displaying moderate activity. Of note, among the mixed gold(I) NHC-alkynyl compounds, one features a fluorescent boron-dipyrromethene (BODIPY) moiety which allowed determining its uptake into the cytoplasm of cancer cells by fluorescence microscopy. 


\section{INTRODUCTION}

The medicinal use of gold is known since ancient Egyptian times and in the $17^{\text {th }}$ century colloidal gold was applied for treatment of fever and sickness [1,2]. At the beginning of the 1900s, Koch and co-workers described the bacteriostatic activity of $\mathrm{K}\left[\mathrm{Au}(\mathrm{CN})_{2}\right]$, and 30 years later, a gold(I) phosphane-thiolate complex, Auranofin (Fig. 1), was recognised as an anti-rheumatic metallopharmaceutical capable of reducing inflammation and the progression of this chronic disease [3]. Subsequently, Auranofin has been evaluated in vitro for its anticancer properties [4,5]. It is nowadays actively being repurposed in pilot trials and clinical studies for anticancer therapy against chronic lymphocytic leukaemia and ovarian cancer among others [6,7].

Potential gold-based pharmaceuticals are prepared in the oxidation states $+1 /+3$. In both redox states, gold complexes can be easily reduced to colloidal gold, which leads to metallodrug inactivation or to unselective cellular damage. In order to achieve the control of the metal complex's reactivity and speciation in biological environment, organometallic compounds, featuring a direct metal-carbon bond which further stabilises the metal centre, have been widely explored in medicinal chemistry [8,9]. In this context, various families of gold(I) and gold(III) organometallic compounds have been recently designed as a source of stable and biologically active molecules [9-11]. For example, gold(I) $\mathrm{N}$-heterocyclic carbenes (NHCs) are of particular interest since the strong electron-donating properties of the carbene ligand stabilises the gold(I) centre against reduction, even under physiological conditions [10,12]. Moreover, the NHC core structure can be chemically modified conferring different physicochemical and functional properties to the resulting metallopharmaceutical. Thus, since their first report, several gold(I) NHC complexes have been shown to possess promising cytotoxic properties against cancer cells, or even antibacterial effects [13-24]. The proposed mechanism of action of this class of oganometallic complexes has been linked to their affinity for binding to thiol and selenol groups in protein targets. For example, the selenoenzyme thioredoxin reductase (TrxR), involved in maintaining the redox homeostasis in cells, has 
been shown to be efficiently inhibited by gold(I)/gold(III) complexes [25-27], including gold(I) NHCs $[21,28-30]$.

In the last years, metal NHCs complexes derived from xanthines, including of platinum(II) and silver(I), have attracted much attention for therapeutic applications. In fact, the easy functionalization of xanthines, combined with their biological activity, makes their metal derivatives excellent candidates for the development of novel metallodrugs[31]. Recently, we reported on a cationic gold(I) bis-NHC complex $\left[\mathrm{Au}(9-\text { methylcaffein-8-ylidene) }]^{+}(\mathbf{A u T M X} \mathbf{2}\right.$, Fig. 1) that is selectively cytotoxic against the ovarian cancer cell line A2780. Moreover, the compound was reported to selectively stabilize human telomeric (hTelo) G-quadruplexes (G4s), a type of DNA secondary structure present in the telomeric regions of the chromosomes [32]. G4s are non-canonical DNA secondary structures formed in guanine rich sequences and have been identified in human telomeres and promoter regions of many genes. They are supposed to regulate important biological processes such as telomere homeostasis, gene transcription and DNA replication [33-35]. Stabilizing these DNA secondary structures by small molecules may interfere with their physiological function and induce potential anticancer effects, e.g. by inhibiting telomere extensions or oncogene expression [34-36].

Initial structure activity relationship studies revealed that any modification at the N9-position of the xanthine in the AuTMX 2 scaffold leads to a loss of G4 binding potency and selectivity. From X-ray diffraction analysis, it was found that any bulky modification at the N9-position of the xanthine scaffold would force the two carbenes ligands coordinating the gold(I) centre out of planarity, probably reducing the strength of the interaction with the flat aromatic hTelo-G4 surface. This was also corroborated by experimental evidences in solution [37]. Furthermore, via a combination of computational and experimental assays, the binding energy of AuTMX2 with hTelo was elucidated [37].

In the present study, we reasoned that modifications at the N1-position of the xanthine ligand would retain the planar geometry of the resulting gold(I) bis-NHCs, constituting a promising path for 
obtaining further structure-activity relationships for this class of G4-stabilising compounds. Therefore, eight new complexes were synthesized and characterized by different techniques. Next to four cationic N1-modified AuTMX2 analogues $\left[\mathrm{Au}(\mathrm{N} 1-\mathrm{TBM})_{2}\right] \mathrm{BF}_{4}(\mathrm{~N} 1-\mathrm{TBM}=\mathrm{N}-1$ substituted 9methyltheobromin-8-ylidene), we explored the effect of replacing one of the NHC ligands with an alkynyl moiety on the G4s stabilizing potency in the new series of general formula [Au(N1TBM)alkynyl]. It should be noted that the latter family of compounds is neutral. In a previous work, a mixed NHC-alkynyl gold(I) complex showed potent cytotoxic activity in human cancer cells while being scarcely toxic in healthy rat liver tissues ex vivo [20]. Thus, four gold(I) organometallics featuring mixed NHC-alkynyl ligands were also synthesized and characterized by different methods. The stability of all the new compounds was studied in aqueous environment and in the presence of N-acethyl-cysteine (NAC), used as model nucleophile, by NMR spectroscopy. Afterward, the two families were comparatively evaluated with respect to their G4-stabilization in vitro. It should be noted that, since the G4-stabilization of AuTMX2 was previously assessed only on telomeric G4s, the present study evaluates the new gold(I) compounds towards both telomeric (hTelo) and promoter G4s (C-KIT1 and hTERT) by fluorescence resonance energy transfter (FRET) DNA melting, and in selected cases also by circular dichroism (CD). Finally, the cytotoxic properties of the compounds have been studied in a small panel of human cancer cells in vitro, and one of the derivatives, endowed with fluorescence properties, also charcaterized for its uptake in melanoma cells by fluorescence microscopy.

Figure 1.

\section{MATERIALS AND METHODS}

\section{General.}

Solvents and reagents (reagent grade) were all commercially available and used without further purification. ${ }^{1} \mathrm{H},{ }^{11} \mathrm{~B}$ and ${ }^{13} \mathrm{C}$ NMR spectra were recorded in $\mathrm{CD}_{3} \mathrm{CN}$ (deuterated acetonitrile), $\mathrm{CDCl}_{3}$ (detuterated chloroform), DMSO- $d_{6}$ (deuterated dimethyl sulfoxide) or acetone- $d_{6}$ solution, with 
tetramethylsilane (TMS) as an internal reference, on a Bruker Avance NMR spectrometer (300-500 MHz, Fig. S1-S33). Electrospray ionization (ESI) mass spectra were recorded on a Synapt G2-Si time-of-flight mass spectrometer (Waters Corporation, Milford, USA). Elemental analysis were performed by the Microanalytical Laboratory at the Technical University of Munich. Au(tht)Cl (tht = tetrahydrothiophene) and AuTMX2, AuTMXI ( [Au(9-methylcaffein-8-ylidene)Iodide], Fig. 1), and the BODIPY-alkynyl ligand were synthesised according procedures already published in literature $[37,38]$.

\section{Synthesis and characterisation.}

1-Ethyl-3,7-dimethylxanthine (1a): Theobromine $(200 \mathrm{mg}, 1.1 \mathrm{mmol})$ and potassium carbonate (228 mg, $2.4 \mathrm{mmol}$ ) were suspended in dimethylformamide (DMF, $4 \mathrm{~mL}$ ) in a round bottom flask. $30 \mathrm{~min}$ later, iodoethane $(176 \mu \mathrm{L}, 1.6 \mathrm{mmol})$ was added dropwise and the reaction stirred overnight at room temperature. The resulting suspension was filtered to achieve a clear solution which was completely evaporated. The off-white solid was washed 3 times with isopropanol to obtain the product as a white solid (150 $\mathrm{mg}, 65 \%)$.

${ }^{1} \mathrm{H}$ NMR (400 MHz, $\left.\mathrm{CDCl}_{3}\right) \delta 7.43(\mathrm{~s}, 1 \mathrm{H}, \mathrm{Ar}), 4.02\left(\mathrm{q}, J=7.1 \mathrm{~Hz}, 2 \mathrm{H}, \mathrm{CH}_{2}\right), 3.92(\mathrm{~d}, J=0.6 \mathrm{~Hz}$, $\left.3 \mathrm{H}, \mathrm{CH}_{3}\right), 3.51\left(\mathrm{~s}, 3 \mathrm{H}, \mathrm{CH}_{3}\right), 1.19\left(\mathrm{t}, J=7.1, \mathrm{~Hz}, 3 \mathrm{H}, \mathrm{CH}_{3}\right)$.

${ }^{13} \mathrm{C}$ NMR (101 MHz, DMSO-d $\left.d_{6}\right) \delta 154.71$ (Ar), 151.13 (Ar), 148.68 (Ar), 143.36 (Ar), 107.17 (Ar), $35.99\left(\mathrm{CH}_{2}\right), 33.64\left(\mathrm{CH}_{3}\right), 29.81\left(\mathrm{CH}_{3}\right), 13.60\left(\mathrm{CH}_{3}\right)$.

1-(2-Cyclohexylethyl)-3,7-dimethylxanthine (1b): Theobromine $\quad(200 \mathrm{mg}, \quad 1.1 \mathrm{mmol})$ and potassium carbonate $(500 \mathrm{mg}, 3.6 \mathrm{mmol})$ were suspended in DMF $(8 \mathrm{~mL})$ for $30 \mathrm{~min}$ prior to the addition of (2-bromoethyl)cyclohexane $(208 \mu \mathrm{L}, 1.3 \mathrm{mmol})$. The reaction mixture was heated to reflux overnight. The day after the suspension was filtered off and the resulting clear yellow solution was dried to achieve a sticky solid. A white solid was isolated after recrystallization in isopropanol (240 mg, 75\%). 
${ }^{1} \mathrm{H}$ NMR (400 MHz, DMSO-d $) \delta 7.99(\mathrm{~s}, 1 \mathrm{H}, \mathrm{Ar}), 3.86\left(\mathrm{~s}, 3 \mathrm{H}, \mathrm{CH}_{3}\right), 3.39\left(\mathrm{~s}, 3 \mathrm{H}, \mathrm{CH}_{3}\right), 1.66(\mathrm{dt}$, $\left.J=28.2,15.6 \mathrm{~Hz}, 6 \mathrm{H}, \mathrm{CH}_{2}\right), 1.43-1.36\left(\mathrm{~m}, 2 \mathrm{H}, \mathrm{CH}_{2}\right), 1.34-1.05\left(\mathrm{~m}, 5 \mathrm{H}, \mathrm{CH}\right.$ and $\left.\mathrm{CH}_{2}\right), 0.97-0.83$ (m, 2H, $\left.\mathrm{CH}_{2}\right)$.

${ }^{13} \mathrm{C}$ NMR (101 MHz, DMSO-d $) \delta 154.77$ (Ar), 151.19 (Ar), 148.62 (Ar), 143.33 (Ar), 107.11 (Ar), $35.66\left(\mathrm{CH}_{2}\right), 35.41\left(\mathrm{CH}_{2}\right), 33.61\left(\mathrm{CH}_{3}\right), 33.39\left(\mathrm{CH}_{2}\right), 33.13\left(\mathrm{CH}_{2}\right), 29.81\left(\mathrm{CH}_{3}\right), 26.53\left(\mathrm{CH}_{2}\right), 26.13$ $\left(\mathrm{CH}_{2}\right)$.

Unfortunately, the synthesis of this compound was succesfull only once and could not be reproduced to perform complete characterization by mass spectrometry and elemental analysis.

1-Benzyl-3,7-dimethylxanthine (1c): Theobromine (200 mg, $1.1 \mathrm{mmol})$ and potassium carbonate (228 mg, $2.4 \mathrm{mmol}$ ) were suspended in DMF (4 mL) in a round bottom flask. 30 min later, benzyl bromide $(142 \mu \mathrm{L}, 1.6 \mathrm{mmol})$, was added dropwise and the reaction stirred overnight at room temperature. The resulting suspension was completely evaporated and water $(20 \mathrm{~mL})$ was added. The product was afterwards extracted with dichloromethane $(\mathrm{DCM}, 3 \times 15 \mathrm{~mL})$, dried over magnesium sulfate and fully evaporated. The resulting product was obtained after recrystallization in methanol (268 mg, 89\%).

${ }^{1} \mathrm{H}$ NMR (400 MHz, DMSO-d $\left.d_{6}\right) \delta 8.05$ (s, 1H, Ar), 7.29 (t, $\left.J=4.2 \mathrm{~Hz}, 4 \mathrm{H}, \mathrm{Ar}\right), 7.27-7.21(\mathrm{~m}, 1 \mathrm{H}$, Ar), $5.06\left(\mathrm{~s}, 2 \mathrm{H}, \mathrm{CH}_{2}\right), 3.89\left(\mathrm{~s}, 3 \mathrm{H}, \mathrm{CH}_{3}\right), 3.43\left(\mathrm{~s}, 3 \mathrm{H}, \mathrm{CH}_{3}\right)$.

${ }^{13} \mathrm{C}$ NMR (101 MHz, DMSO-d6) $\delta 154.89$ (Ar), 151.45 (Ar), 148.91 (Ar), 143.65 (Ar), 138.09 (Ar), $128.74(\mathrm{Ar}), 127.97(\mathrm{Ar}), 127.50(\mathrm{Ar}), 107.14(\mathrm{Ar}), 44.01\left(\mathrm{CH}_{2}\right), 33.68\left(\mathrm{CH}_{3}\right), 29.97\left(\mathrm{CH}_{3}\right)$.

3,7-Dimethyl-1-(4-trifluoromethyl)benzylxanthine (1d): A suspension of theobromine (360 mg, $2.0 \mathrm{mmol})$ in $\mathrm{DMF}(15 \mathrm{~mL})$ was heated to $50{ }^{\circ} \mathrm{C}$ for $10 \mathrm{~min}$ before adding 3 equiv. of potassium carbonate $(830 \mathrm{mg}, 6 \mathrm{mmol})$. After $30 \mathrm{~min}$, an equimolar amount of 4-(trifluoromethyl)benzyl bromide $(309 \mu \mathrm{L}, 2.0 \mathrm{mmol})$ was added and the reaction mixture stirred overnight at $50{ }^{\circ} \mathrm{C}$. Thus, the 
suspension was filtered off and the resulting clear solution was dried to achieve a white solid. Further recrystallization in isopropanol allowed to isolate the final product as an off-white solid (423 $\mathrm{mg}$, $63 \%)$

${ }^{1} \mathrm{H}$ NMR $\left(300 \mathrm{MHz}, \mathrm{CDCl}_{3}\right) \delta 7.65-7.49(\mathrm{~m}, 4 \mathrm{H}, \mathrm{Ar}), 5.25\left(\mathrm{~s}, 2 \mathrm{H}, \mathrm{CH}_{2}\right), 4.01\left(\mathrm{~s}, 3 \mathrm{H}, \mathrm{CH}_{3}\right), 3.60(\mathrm{~s}$, $\left.3 \mathrm{H}, \mathrm{CH}_{3}\right)$.

${ }^{13} \mathrm{C}$ NMR (101 MHz, $\left.\mathrm{CDCl}_{3}\right) \delta 154.12$ (Ar), 150.54 (Ar), 148.03 (Ar), 140.75 (Ar), 140.19 (Ar), $128.74\left(\mathrm{q}, J=32.32 \mathrm{~Hz}, \mathrm{Ar}, \mathrm{C}_{\alpha}-\mathrm{CF}_{3}\right), 128.02(\mathrm{Ar}), 126.03\left(\mathrm{q}, J=272.7 \mathrm{~Hz}, \mathrm{CF}_{3}\right), 124.37(\mathrm{q}, J=$ 3.8 Hz, Ar, $\left.\mathrm{C}_{\beta}-\mathrm{CF}_{3}\right), 106.59(\mathrm{Ar}), 43.02\left(\mathrm{CH}_{2}\right), 32.62\left(\mathrm{CH}_{3}\right), 28.82\left(\mathrm{CH}_{3}\right)$.

1-Ethyl-3,7,9-trimethylxanthinium tetrafluoroborate (2a): Compound 1a (214 mg, $1.0 \mathrm{mmol})$ was dissolved in acetonitrile $(\mathrm{MeCN}, 8 \mathrm{~mL})$ prior to the addition of trimethyl-oxonium tetrafluoroborate $(243 \mathrm{mg}, 1.6 \mathrm{mmol})$. After $5 \mathrm{~min}$, sodium carbonate (200 mg, $1.9 \mathrm{mmol}$ ) was added and the mixture stirred for $1 \mathrm{~h}$ at room temperature under an inert atmosphere. The resulting suspension was filtered to achieve a clear solution which was further dried. The solid was washed with $\mathrm{Et}_{2} \mathrm{O}$ and DCM to afford a sticky solid. The final white solid was obtained after recrystallization in isopropanol (226 mg, 74\%).

${ }^{1} \mathrm{H}$ NMR (400 MHz, CD $\left.3 \mathrm{CN}\right) \delta 8.47(\mathrm{~s}, 1 \mathrm{H}, \mathrm{Ar}), 4.08\left(\mathrm{~s}, 6 \mathrm{H}, \mathrm{CH}_{3}\right), 4.02\left(\mathrm{q}, J=7.1 \mathrm{~Hz}, 2 \mathrm{H}, \mathrm{CH}_{2}\right)$, $3.73\left(\mathrm{~s}, 3 \mathrm{H}, \mathrm{CH}_{3}\right), 1.21\left(\mathrm{t}, J=7.1 \mathrm{~Hz}, 3 \mathrm{H}, \mathrm{CH}_{3}\right)$.

${ }^{13} \mathrm{C}$ NMR (126 MHz, CD 3 CN) $\delta 154.21$ (Ar), 151.02 (Ar), 140.55 (Ar), 139.58 (Ar), 109.48 (Ar), $38.16\left(\mathrm{CH}_{3}\right), 37.86\left(\mathrm{CH}_{3}\right), 36.59\left(\mathrm{CH}_{2}\right), 31.99\left(\mathrm{CH}_{3}\right), 12.92\left(\mathrm{CH}_{3}\right)$.

Elemental analysis for $\mathrm{C}_{10} \mathrm{H}_{19} \mathrm{BF}_{4} \mathrm{~N}_{4} \mathrm{O}_{4}\left(\mathbf{2 a} \cdot 2 \mathrm{H}_{2} \mathrm{O}\right)$ : C $35.02 \%$, H 5.68\%, N $16.37 \%$ (calc. C 34.70\%, H $5.53 \%, \mathrm{~N} 16.19 \%)$

1-(2-Cyclohexylethyl)-3,7,9-trimethylxanthinium tetrafluoroborate $\quad(2 \mathrm{~b}):$ Compound $1 \mathrm{~b}$ (500 mg, $1.7 \mathrm{mmol}$ ) was dissolved in $\mathrm{MeCN}(7 \mathrm{~mL})$ prior to the addition of trimethyloxonium tetrafluoroborate (267 mg, $1.8 \mathrm{mmol})$. After $5 \mathrm{~min}$, sodium carbonate (288 mg, $2.7 \mathrm{mmol}$ ) was added 
and stirred for $4 \mathrm{~h}$ at $40{ }^{\circ} \mathrm{C}$ under an inert atmosphere. The resulting suspension was filtered to achieve a clear solution which was further dried. A final recrystallization in isopropanol allowed to obtain the final product as a white solid (304 $\mathrm{mg}, 89 \%)$.

${ }^{1} \mathrm{H}$ NMR $\left(400 \mathrm{MHz}, \mathrm{CD}_{3} \mathrm{CN}\right) \delta 8.43(\mathrm{~s}, 1 \mathrm{H}, \mathrm{Ar}), 4.05\left(\mathrm{t}, J=2.6 \mathrm{~Hz}, 6 \mathrm{H}, \mathrm{CH}_{3}\right), 3.99-3.93(\mathrm{~m}, 2 \mathrm{H}$, $\left.\mathrm{CH}_{2}\right), 3.69\left(\mathrm{~s}, 3 \mathrm{H}, \mathrm{CH}_{3}\right), 1.83-1.61\left(\mathrm{~m}, 5 \mathrm{H}, \mathrm{CH}\right.$ and $\left.\mathrm{CH}_{2}\right), 1.50-1.42\left(\mathrm{~m}, 2 \mathrm{H}, \mathrm{CH}_{2}\right), 1.38-1.13$ (m, 4H, $\left.\mathrm{CH}_{2}\right), 1.03-0.90\left(\mathrm{~m}, 2 \mathrm{H}, \mathrm{CH}_{2}\right)$.

${ }^{13} \mathrm{C}$ NMR (101 MHz, CD 3 CN) $\delta 154.37$ (Ar), 151.16 (Ar), 140.57 (Ar), 139.53 (Ar), 109.50 (Ar), $41.25\left(\mathrm{CH}_{2}\right), 37.94\left(\mathrm{CH}_{3}\right), 36.68\left(\mathrm{CH}_{2}\right), 36.41\left(\mathrm{CH}_{3}\right), 35.58\left(\mathrm{CH}_{2}\right), 33.82\left(\mathrm{CH}_{2}\right), 32.09\left(\mathrm{CH}_{3}\right), 27.20$ $\left(\mathrm{CH}_{2}\right), 26.93\left(\mathrm{CH}_{2}\right)$.

Unfortunately, due to the impossibility to reproduce the synthesis of compound $\mathbf{1 b}$ (see above), this compound was not obtained in sufficient quantity to perform complete characterization by mass spectrometry and elemental analysis.

1-Benzyl-3,7,9-trimethylxanthinium tetrafluoroborate (2c): Compound 1c (273 mg, $1.0 \mathrm{mmol})$ was dissolved in $\mathrm{MeCN}(8 \mathrm{~mL})$ prior to the addition of trimethyloxonium tetrafluoroborate $(243 \mathrm{mg}$, $1.6 \mathrm{mmol})$. After $5 \mathrm{~min}$, sodium carbonate $(288 \mathrm{mg}, 2.7 \mathrm{mmol})$ was added and stirred for $4 \mathrm{~h}$ at $40{ }^{\circ} \mathrm{C}$ under an inert atmosphere. The resulting suspension was filtered to achieve a clear solution which was further dried. The solid was washed with $\mathrm{Et}_{2} \mathrm{O}$ and DCM. Recrystallization from isopropanol yielded a white solid (357 mg, 93\%).

${ }^{1} \mathrm{H}$ NMR (400 MHz, CD $\left.3 \mathrm{CN}\right) \delta 8.48(\mathrm{~s}, 1 \mathrm{H}, \mathrm{Ar}), 7.47-7.24(\mathrm{~m}, 5 \mathrm{H}, \mathrm{Ar}), 5.16\left(\mathrm{~s}, 2 \mathrm{H}, \mathrm{CH}_{2}\right), 4.14-$ $4.02\left(\mathrm{~m}, 6 \mathrm{H}, \mathrm{CH}_{3}\right), 3.73\left(\mathrm{~s}, 3 \mathrm{H}, \mathrm{CH}_{3}\right)$.

${ }^{13} \mathrm{C}$ NMR (101 MHz, CD 3 CN) $\delta 154.09$ (Ar), 150.97 (Ar), 140.40 (Ar), 139.35 (Ar), 137.10 (Ar), $129.04(\mathrm{Ar}), 128.57(\mathrm{Ar}), 128.23(\mathrm{Ar}), 109.14(\mathrm{Ar}), 45.56\left(\mathrm{CH}_{2}\right), 37.59\left(\mathrm{CH}_{3}\right), 36.36\left(\mathrm{CH}_{3}\right), 31.87$ $\left(\mathrm{CH}_{3}\right)$.

Elemental analysis for $\mathrm{C}_{15} \mathrm{H}_{25} \mathrm{BF}_{4} \mathrm{~N}_{4} \mathrm{O}_{6}\left(2 \mathrm{c} \cdot 4 \mathrm{H}_{2} \mathrm{O}\right)$ : C $40.73 \%$, H 5.41\%, N $12.55 \%$ (calc. C 40.56\%, H $5.67 \%$, N $12.61 \%)$ 
3,7,9-Trimethyl-1-(4-trifluoromethyl)benzylxanthinium tetrafluoroborate (2d): Compound 1d (202 $\mathrm{mg}, 0.6 \mathrm{mmol})$ was dissolved in $\mathrm{MeCN}(6 \mathrm{~mL})$ prior to the addition of trimethyloxonium tetrafluoroborate (262 mg, $1.8 \mathrm{mmol})$. After $5 \mathrm{~min}$, sodium carbonate ( $288 \mathrm{mg}, 2.7 \mathrm{mmol}$ ) was added and stirred for $5 \mathrm{~h}$ at $50{ }^{\circ} \mathrm{C}$ under an inert atmosphere. The resulting suspension was filtered to achieve a clear solution which was further dried. The final white solid was obtained after recrystallization in isopropanol (154.7 $\mathrm{mg}, 62 \%)$.

${ }^{1} \mathrm{H}$ NMR (400 MHz, CD $\left.3 \mathrm{CN}\right) \delta 8.47(\mathrm{~s}, 1 \mathrm{H}, \mathrm{Ar}), 7.63(\mathrm{dd}, J=45.5,8.1 \mathrm{~Hz}, 4 \mathrm{H}, \mathrm{Ar}), 5.23(\mathrm{~s}, 2 \mathrm{H}$, $\left.\mathrm{CH}_{2}\right), 4.09$ (s, 3H, $\left.\mathrm{CH}_{3}\right), 4.08$ (s, 3H, $\left.\mathrm{CH}_{3}\right), 3.74$ (s, 3H, $\left.\mathrm{CH}_{3}\right)$.

${ }^{13} \mathrm{C}$ NMR (101 MHz, CD $\left.3 \mathrm{CN}\right) \delta 154.06$ (Ar), 150.97 (Ar), 141.62 (Ar), 140.49 (Ar), 139.50 (Ar), $129.69\left(\mathrm{q}, J=32.32 \mathrm{~Hz}, \mathrm{Ar}, \mathrm{C}_{\alpha}-\mathrm{CF}_{3}\right), 125.88\left(\mathrm{q}, J=3.0 \mathrm{~Hz}, \mathrm{Ar}, \mathrm{C}_{\beta}-\mathrm{CF}_{3}\right), 125.25$ (q, $J=272.7 \mathrm{~Hz}$, $\left.\mathrm{CF}_{3}\right), 125.88(\mathrm{Ar}), 125.84(\mathrm{Ar}), 123.55(\mathrm{Ar}), 109.15(\mathrm{Ar}), 45.20\left(\mathrm{CH}_{2}\right), 37.63\left(\mathrm{CH}_{3}\right), 36.37\left(\mathrm{CH}_{3}\right)$, $31.93\left(\mathrm{CH}_{3}\right)$.

Elemental analysis for $\mathrm{C}_{16} \mathrm{H}_{20} \mathrm{BF}_{7} \mathrm{~N}_{4} \mathrm{O}_{4}\left(\mathbf{2 d} \cdot 2 \mathrm{H}_{2} \mathrm{O}\right)$ : C 40.14\%, H 4.17\%, N $11.43 \%$ (calc. C 40.36\%, $\mathrm{H} 4.23 \%, \mathrm{~N} 11.77 \%$ )

Bis(1-ethyl-3,7,9-trimethylxanthin-8-ylidene)gold(I) tetrafluoroborate (3a): Ligand 2a (100 mg, $0.32 \mathrm{mmol})$ and $\mathrm{Ag}_{2} \mathrm{O}(59.79 \mathrm{mg}, 0.26 \mathrm{mmol})$ were suspended in dry acetonitrile $(10 \mathrm{~mL})$ with molecular sieves $(4 \AA)$ under an inert atmosphere. After $6 \mathrm{~h}$ at room temperature, $\mathrm{Au}(\mathrm{tht}) \mathrm{Cl}(51.7 \mathrm{mg}$, $0.16 \mathrm{mmol}$ ) was added and the suspension stirred overnight. The day after potassium iodide (27 mg, $0.16 \mathrm{mmol}$ ) was added and the mixture was left under vigorous stirring for further $30 \mathrm{~min}$. The final dark grey suspension was filtered over Celite ${ }^{\circledR}$ and washed with acetonitrile and DCM to give a clear colourless solution. After concentration to ca. $1 \mathrm{~mL}$, an excess of $\mathrm{Et}_{2} \mathrm{O}$ was added to precipitate the final white solid (46 mg, 39\%). 
${ }^{1} \mathrm{H}$ NMR $\left(400 \mathrm{MHz}, \mathrm{CD}_{3} \mathrm{CN}\right) \delta 4.22\left(\mathrm{~s}, 3 \mathrm{H}, \mathrm{CH}_{3}\right), 4.19\left(\mathrm{~s}, 3 \mathrm{H}, \mathrm{CH}_{3}\right), 4.03\left(\mathrm{~d}, J=6.9 \mathrm{~Hz}, 2 \mathrm{H}, \mathrm{CH}_{2}\right)$, $3.77\left(\mathrm{~s}, 3 \mathrm{H}, \mathrm{CH}_{3}\right), 1.21\left(\mathrm{t}, J=7.0 \mathrm{~Hz}, 3 \mathrm{H}, \mathrm{CH}_{3}\right)$.

${ }^{13} \mathrm{C}$ NMR (126 MHz, CD 3 CN) $\delta 188.37$ (Ar), 154.35 (Ar), 151.45 (Ar), 141.62 (Ar), 110.28 (Ar), $39.70\left(\mathrm{CH}_{3}\right), 38.04\left(\mathrm{CH}_{3}\right), 37.91\left(\mathrm{CH}_{2}\right), 32.30\left(\mathrm{CH}_{3}\right), 13.12\left(\mathrm{CH}_{3}\right)$.

ESI-MS $\left(\mathrm{CH}_{3} \mathrm{CN}\right.$, pos. mode) for $\mathrm{C}_{20} \mathrm{H}_{27} \mathrm{AuN}_{8} \mathrm{O}_{4}$ : exp. 641.1909 (calc. 641.1875).

Elemental analysis for $\mathrm{C}_{20} \mathrm{H}_{32} \mathrm{AuBF}_{4} \mathrm{~N}_{8} \mathrm{O}_{6}\left(3 \mathbf{a} \cdot 2 \mathrm{H}_{2} \mathrm{O}\right.$ ): exp. C $31.87 \%$, H 4.34\%, N $14.33 \%$ (calc. C $31.43 \%, \mathrm{H} 4.22 \%, \mathrm{~N} 14.66 \%)$

\section{Bis(1-(2-cyclohexylethyl)-3,7,9-trimethylxanthin-8-ylidene)gold(I) tetrafluoroborate (3b):}

Ligand 2b (133 mg, $0.32 \mathrm{mmol})$ and $\mathrm{Ag}_{2} \mathrm{O}(67 \mathrm{mg}, 0.29 \mathrm{mmol})$ were suspended in dry $\mathrm{MeCN}$ (10 mL) with molecular sieves $(4 \AA)$ under an inert atmosphere. After $6 \mathrm{~h}$ at room temperature, $\mathrm{Au}(\mathrm{tht}) \mathrm{Cl}(81 \mathrm{mg}, 0.25 \mathrm{mmol})$ was added and the suspension stirred overnight. Thus, potassium iodide (42 $\mathrm{mg}, 0.25 \mathrm{mmol}$ ) was added and the mixture was left under vigorous stirring for further 30 min. The final dark grey suspension was filtered over Celite ${ }^{\circledR}$ and washed with $\mathrm{MeCN}$ and DCM to give a clear colourless solution. After concentration to ca. $1 \mathrm{~mL}$, an excess of $\mathrm{Et}_{2} \mathrm{O}$ was added to precipitate the final white compound $(57 \mathrm{mg}, 38 \%)$.

${ }^{1} \mathrm{H}$ NMR (400 MHz, CD $\left.{ }_{3} \mathrm{CN}\right) \delta 4.19\left(\mathrm{~s}, 3 \mathrm{H}, \mathrm{CH}_{3}\right), 4.15\left(\mathrm{~s}, 3 \mathrm{H}, \mathrm{CH}_{3}\right), 4.13\left(\mathrm{~s}, 3 \mathrm{H}, \mathrm{CH}_{3}\right), 4.04(\mathrm{~s}, 3 \mathrm{H}$, $\left.\mathrm{CH}_{3}\right), 3.95$ (ddd, $\left.J=9.8,7.8,5.5 \mathrm{~Hz}, 4 \mathrm{H}, \mathrm{CH}_{2}\right), 3.73\left(\mathrm{~s}, 3 \mathrm{H}, \mathrm{CH}_{3}\right), 3.69\left(\mathrm{~s}, 3 \mathrm{H}, \mathrm{CH}_{3}\right), 1.82-1.60(\mathrm{~m}$, 10H, $\mathrm{CH}$ and $\left.\mathrm{CH}_{2}\right), 1.50-1.40\left(\mathrm{~m}, 4 \mathrm{H}, \mathrm{CH}_{2}\right), 1.37-1.10\left(\mathrm{~m}, 9 \mathrm{H}, \mathrm{CH}_{2}\right), 1.03-0.89\left(\mathrm{~m}, 4 \mathrm{H}, \mathrm{CH}_{2}\right)$. ${ }^{13} \mathrm{C}$ NMR (126 MHz, CD $\left.3 \mathrm{CN}\right) \delta 188.34$ (Ar), 176.95 (Ar), 154.46 (Ar), 154.42 (Ar), 151.53 (Ar), 141.60 (Ar), 141.16 (Ar), 110.25 (Ar), 109.71 (Ar), 109.24 (Ar), 40.98 (Ar), $40.90\left(\mathrm{CH}_{3}\right), 39.82$ $\left(\mathrm{CH}_{3}\right), 39.70\left(\mathrm{CH}_{2}\right), 38.16\left(\mathrm{CH}_{2}\right), 38.05\left(\mathrm{CH}_{2}\right), 36.47\left(\mathrm{CH}_{2}\right), 35.75\left(\mathrm{CH}_{2}\right), 33.84\left(\mathrm{CH}_{3}\right), 32.33(\mathrm{CH}$ and $\left.\mathrm{CH}_{3}\right), 27.20\left(\mathrm{CH}_{3}\right), 26.92\left(\mathrm{CH}_{3}\right)$.

Unfortunately, due to the impossibility to reproduce the synthesis of ligand $\mathbf{2 b}$ (see above), this compound was not obtained in sufficient quantity to perform complete characterization by mass spectrometry and elemental analysis. 
Bis(1-benzyl-3,7,9-trimethylxanthin-8-ylidene)gold(I) tetrafluoroborate $\quad(3 c)$ : Ligand 2c (159 mg, $0.42 \mathrm{mmol})$ and $\mathrm{Ag}_{2} \mathrm{O}(80 \mathrm{mg}, 0.35 \mathrm{mmol})$ were suspended in dry $\mathrm{MeCN}(10 \mathrm{~mL})$ with molecular sieves $(4 \AA)$ under an inert atmosphere. After $6 \mathrm{~h}$ at room temperature, Au(tht)Cl (89 mg, $0.28 \mathrm{mmol}$ ) was added and the suspension stirred overnight. Thus, potassium iodide (48 $\mathrm{mg}$, $0.28 \mathrm{mmol}$ ) was added and the mixture was left under vigorous stirring for further $30 \mathrm{~min}$. The final dark grey suspension was filtered over Celite ${ }^{\circledR}$ and washed with acetonitrile and DCM to give a clear colourless solution. After concentration to ca. $1 \mathrm{~mL}$, an excess of $\mathrm{Et}_{2} \mathrm{O}$ was added to precipitate out the final white compound (34 mg, 20\%).

${ }^{1} \mathrm{H}$ NMR (400 MHz, CD $\left.3 \mathrm{CN}\right) \delta 7.34-7.15$ (m, 5H, Ar), $5.06\left(\mathrm{~s}, 2 \mathrm{H}, \mathrm{CH}_{2}\right), 4.12\left(\mathrm{~s}, 3 \mathrm{H}, \mathrm{CH}_{3}\right), 4.08$ (s, $\left.3 \mathrm{H}, \mathrm{CH}_{2}\right), 3.67\left(\mathrm{~s}, 3 \mathrm{H}, \mathrm{CH}_{3}\right)$.

${ }^{13} \mathrm{C}$ NMR (101 MHz, CD 3 CN) $\delta 188.61$ (Ar), 154.57 (Ar), 151.76 (Ar), 141.85 (Ar), 137.98 (Ar), 129.36 (Ar), 128.89 (Ar), $128.45(\mathrm{Ar}), 110.27(\mathrm{Ar}), 45.72\left(\mathrm{CH}_{2}\right), 39.76\left(\mathrm{CH}_{3}\right), 38.11\left(\mathrm{CH}_{3}\right), 32.50$ $\left(\mathrm{CH}_{3}\right)$.

Elemental analysis for $\mathrm{C}_{30} \mathrm{H}_{36} \mathrm{AuBF}_{4} \mathrm{~N}_{8} \mathrm{O}_{6}\left(3 \mathbf{c} \cdot 2 \mathrm{H}_{2} \mathrm{O}\right.$ ): exp. C 40.34\%, H 3.75\%, N 12.90\% (calc. C $40.56 \%, \mathrm{H} 4.08 \%, \mathrm{~N} 12.61 \%)$

\section{Bis(3,7,9-trimethyl-1-(4-trifluoromethyl)benzylxanthin-8-ylidene)gold(I) tetrafluoroborate}

(3d): Ligand 2d (130 mg, $0.30 \mathrm{mmol})$ and $\mathrm{Ag}_{2} \mathrm{O}$ (54.8 mg, $\left.0.24 \mathrm{mmol}\right)$ were suspended in dry MeCN (10 mL) with molecular sieves $(4 \AA)$ under inert atmosphere. After $6 \mathrm{~h}$ at room temperature, $\mathrm{Au}(\mathrm{tht}) \mathrm{Cl}(48 \mathrm{mg}, 0.15 \mathrm{mmol})$ was added and the suspension stirred overnight. Thus, potassium iodide ( $25 \mathrm{mg}, 0.15 \mathrm{mmol}$ ) was added and the mixture was left under vigorous stirring for further 30 min. The final dark grey suspension was filtered over Celite ${ }^{\circledR}$ and washed with acetonitrile and DCM to give a clear colourless solution. After concentration to ca. $1 \mathrm{~mL}$, an excess of $\mathrm{Et}_{2} \mathrm{O}$ was added to precipitate out the final white compound (100 mg, 66\%). 
${ }^{1} \mathrm{H}$ NMR $\left(500 \mathrm{MHz}, \mathrm{CD}_{3} \mathrm{CN}\right) \delta 7.60(\mathrm{dd}, J=52.1,8.1 \mathrm{~Hz}, 2 \mathrm{H}, \mathrm{Ar}), 5.20\left(\mathrm{~s}, 2 \mathrm{H}, \mathrm{CH}_{2}\right), 4.21(\mathrm{~s}, 3 \mathrm{H}$, $\left.\mathrm{CH}_{3}\right), 4.16\left(\mathrm{~s}, 3 \mathrm{H}, \mathrm{CH}_{3}\right), 3.75\left(\mathrm{~s}, 3 \mathrm{H}, \mathrm{CH}_{3}\right)$.

${ }^{13} \mathrm{C}$ NMR (126 MHz, CD 3 CN) $\delta 188.75$ (Ar), 154.52 (Ar), 151.76 (Ar), 142.53 (Ar), 141.96 (Ar), $129.94\left(\mathrm{q}, J=32.76 \mathrm{~Hz}, \mathrm{Ar}, \mathrm{C}_{\alpha}-\mathrm{CF}_{3}\right), 129.51(\mathrm{Ar}), 126.23\left(\mathrm{q}, J=3.8 \mathrm{~Hz}, \mathrm{Ar}, \mathrm{C}_{\beta}-\mathrm{CF}_{3}\right), 125.32(\mathrm{q}$, $\left.J=270.9 \mathrm{~Hz}, \mathrm{CF}_{3}\right) 110.29(\mathrm{Ar}), 45.40\left(\mathrm{CH}_{2}\right), 39.80\left(\mathrm{CH}_{3}\right), 38.14\left(\mathrm{CH}_{3}\right), 32.58\left(\mathrm{CH}_{3}\right)$.

ESI-MS ( $\mathrm{CH}_{3} \mathrm{CN}$, pos. mode) for $\mathrm{C}_{32} \mathrm{H}_{30} \mathrm{AuF}_{6} \mathrm{~N}_{8} \mathrm{O}_{4}$ : exp. 901.1976 (calc. 901.1960).

Elemental analysis for $\mathrm{C}_{32} \mathrm{H}_{40} \mathrm{AuBF}_{10} \mathrm{~N}_{8} \mathrm{O}_{9}\left(3 d \cdot \mathrm{H}_{2} \mathrm{O}\right.$ ): C 38.37\%, H 3.13\%, N 11.35\% (calc. C $38.19 \%, \mathrm{H} 3.21 \%, \mathrm{~N} 11.13 \%)$

(Ethynylphenyl)(1,3,7,9-tetramethylxanthin-8-ylidene)gold(I) (4a): Phenylacetylene $(16 \mu \mathrm{L}$, $0.14 \mathrm{mmol})$, was added to $\mathrm{NaOH}(40 \mathrm{mg}, 1 \mathrm{mmol})$ in methanol $(10 \mathrm{~mL})$. After stirring for $15 \mathrm{~min}$ at room temperature, AuTMXI (75 mg, $0.14 \mathrm{mmol}$ ) was added and the mixture stirred under reflux overnight. After filtration, the final product was collected as an off-white solid (50 mg, 69\%).

${ }^{1} \mathrm{H}$ NMR (400 MHz, DMSO-d $\left.d_{6}\right) \delta 7.25(\mathrm{dd}, J=7.8,4.1 \mathrm{~Hz}, 4 \mathrm{H}, \mathrm{Ar}), 7.23-7.16(\mathrm{~m}, 1 \mathrm{H}, \mathrm{Ar}), 4.20$ (s, 3H, $\left.\mathrm{CH}_{3}\right), 4.05$ (s, 3H, $\left.\mathrm{CH}_{3}\right), 3.73\left(\mathrm{~s}, 3 \mathrm{H}, \mathrm{CH}_{3}\right), 3.23\left(\mathrm{~s}, 3 \mathrm{H}, \mathrm{CH}_{3}\right)$.

${ }^{13} \mathrm{C}$ NMR (101 MHz, DMSO-d6) $\delta 190.70$ (Ar), 153.68 (Ar), 150.92 (Ar), 140.82 (Ar), 132.36 (Ar), 131.60 (Ar), 128.67 (Ar), 126.60 (Ar), 126.49 (Ar), 108.80 (C-sp), 104.47 (C-sp), $38.92\left(\mathrm{CH}_{3}\right), 37.33$ $\left(\mathrm{CH}_{3}\right), 31.92\left(\mathrm{CH}_{3}\right), 28.67\left(\mathrm{CH}_{3}\right)$.

ESI-MS ( $\mathrm{CH}_{3} \mathrm{CN}$, pos. mode) for for $\mathrm{C}_{17} \mathrm{H}_{18} \mathrm{AuN}_{4} \mathrm{O}_{2}$ : exp. 507.1096 (calc. 507.1095).

Elemental analysis for $\mathrm{C}_{17} \mathrm{H}_{19} \mathrm{AuN}_{4} \mathrm{O}_{3}\left(4 \mathbf{a} \cdot 1 / 2 \mathrm{H}_{2} \mathrm{O}\right.$ ): exp. C $39.47 \%$, H 3.45\%, N 10.72\% (calc. C $39.62 \%, \mathrm{H} 3.52 \%, \mathrm{~N} 10.87 \%)$

(4b): 4-Ethynylpyridine (16 mg, $0.14 \mathrm{mmol})$ was added to $\mathrm{NaOH}(40 \mathrm{mg}, 1 \mathrm{mmol})$ in methanol $(10 \mathrm{~mL})$. After stirring for 15 
min at room temperature, AuTMXI $(75 \mathrm{mg}, 0.14 \mathrm{mmol})$ was added and the mixture stirred under reflux overnight. After filtration, the final product was collected as an off-white solid (33 mg, 46\%). ${ }^{1} \mathrm{H}$ NMR (400 MHz, DMSO-d6) $\delta 8.41$ (dd, J = 4.5, 1.6 Hz, 2H, Ar), 7.19 (dd, J=4.5, $1.6 \mathrm{~Hz}, 2 \mathrm{H}$, Ar), 4.19 (s, 3H, $\left.\mathrm{CH}_{3}\right), 4.03\left(\mathrm{~s}, 3 \mathrm{H}, \mathrm{CH}_{3}\right), 3.71\left(\mathrm{~s}, 3 \mathrm{H}, \mathrm{CH}_{3}\right), 3.21\left(\mathrm{~s}, 3 \mathrm{H}, \mathrm{CH}_{3}\right)$.

${ }^{13} \mathrm{C}$ NMR (101 MHz, DMSO-d $\left.d_{6}\right) 190.00$ (Ar), 153.67 (Ar), 150.89 (Ar), 149.86 (Ar), 140.81 (Ar), 139.34 (Ar), 133.97 (Ar), 126.06 (Ar), 108.89 (C-sp), 102.18 (C-sp), $38.95\left(\mathrm{CH}_{3}\right), 37.35\left(\mathrm{CH}_{3}\right), 31.91$ $\left(\mathrm{CH}_{3}\right), 28.65\left(\mathrm{CH}_{3}\right)$.

Elemental analysis for $\mathrm{C}_{16} \mathrm{H}_{18} \mathrm{AuN}_{5} \mathrm{O}_{3}\left(\mathbf{4 b} \cdot \mathrm{H}_{2} \mathrm{O}\right)$ : exp. C 36.17\%, H 3.39\%, N 13.07\% (calc. C $36.58 \%, \mathrm{H} 3.45 \%$, N $13.33 \%)$

(3-Ethynylpyridine)(1,3,7,9-tetramethylxanthin-8-ylidene)gold(I) $\quad$ (4c): $\quad 3$-Ethynylpyridine (16 mg, $0.14 \mathrm{mmol})$ was added to $\mathrm{NaOH}(40 \mathrm{mg}, 1 \mathrm{mmol})$ in methanol (10 mL). After stirring for 15 min at room temperature, AuTMXI $(75 \mathrm{mg}, 0.14 \mathrm{mmol})$ was added and the mixture stirred under reflux overnight. After filtration, the final product was collected as an off-white solid (43 mg, 60\%). ${ }^{1} \mathrm{H}$ NMR (400 MHz, DMSO-d6) $\delta 8.46-8.41$ (m, 1H, Ar), 8.36 (dd, $\left.J=4.8,1.5 \mathrm{~Hz}, 1 \mathrm{H}, \mathrm{Ar}\right), 7.65-$ $7.60(\mathrm{~m}, 1 \mathrm{H}, \mathrm{Ar}), 7.27$ (dd, $J=7.9,4.8 \mathrm{~Hz}, 1 \mathrm{H}, \mathrm{Ar}), 4.20\left(\mathrm{~s}, 3 \mathrm{H}, \mathrm{CH}_{3}\right), 4.04\left(\mathrm{~s}, 3 \mathrm{H}, \mathrm{CH}_{3}\right), 3.71$ (s, $\left.3 \mathrm{H}, \mathrm{CH}_{3}\right), 3.20\left(\mathrm{~s}, 3 \mathrm{H}, \mathrm{CH}_{3}\right)$.

${ }^{13} \mathrm{C}$ NMR (101 MHz, DMSO- $d_{6}$ ) $\delta 189.79$ (Ar), 153.20 (Ar), 151.67 (Ar), 150.42 (Ar), 146.47 (Ar), 140.35 (Ar), 137.98 (Ar), 136.01 (Ar), 123.22 (Ar), 122.74 (Ar), 108.37 (C-sp), 100.55 (C-sp), 38.49 $\left(\mathrm{CH}_{3}\right), 36.89\left(\mathrm{CH}_{3}\right), 31.45\left(\mathrm{CH}_{3}\right), 28.20\left(\mathrm{CH}_{3}\right)$.

Elemental analysis for $\mathrm{C}_{16} \mathrm{H}_{18} \mathrm{AuN}_{5} \mathrm{O}_{3}\left(\mathbf{4 c} \cdot \mathrm{H}_{2} \mathrm{O}\right.$ ): exp. C 36.91\%, H 3.21\%, N 13.12\% (calc. C $36.58 \%, \mathrm{H} 3.45 \%, \mathrm{~N} 13.33 \%)$

(2,8-Diethyl-10-(4-ethynylphenyl)-5,5-difluoro-1,3,7,9-tetramethyl-5H-4 $\lambda^{4}, 5 \lambda^{4}$-dipyrrolo[1,2$\left.c: 2^{\prime}, 1^{\prime}-f\right][1,3,2]$ diazaborinine $)(1,3,7,9-t e t r a m e t h y l x a n t h i n-8-y l i d e n e) g o l d(I) \quad(4 d): 2,8-D i e t h y l-$ 
10-(4-ethynylphenyl)-5,5-difluoro-1,3,7,9-tetramethyl-5H-4 $\lambda^{4}, 5 \lambda^{4}$-dipyrrolo[1,2-c:2', $1^{\prime}-$

$f][1,3,2]$ diazaborinine $(55 \mathrm{mg}, 0.14 \mathrm{mmol})$ was added to $\mathrm{NaOH}(40 \mathrm{mg}, 1 \mathrm{mmol})$ in $\mathrm{MeOH}(10 \mathrm{~mL})$. After stirring for $15 \mathrm{~min}$ at room temperature, AuTMXI (75 mg, $0.14 \mathrm{mmol}$ ) was added and the mixture stirred under reflux overnight. After filtration, the final product was collected as a dark red solid. (75.0 mg, 68\%).

${ }^{1} \mathrm{H}$ NMR (400 MHz, acetone- $\left.d_{6}\right) \delta 7.53(\mathrm{~d}, J=8.4 \mathrm{~Hz}, 2 \mathrm{H}, \mathrm{Ar}), 7.26(\mathrm{~d}, J=8.2 \mathrm{~Hz}, 2 \mathrm{H}, \mathrm{Ar}), 4.35$ (s, $\left.3 \mathrm{H}, \mathrm{CH}_{3}\right), 4.13\left(\mathrm{~s}, 3 \mathrm{H}, \mathrm{CH}_{3}\right), 3.91\left(\mathrm{~s}, 3 \mathrm{H}, \mathrm{CH}_{3}\right), 3.31\left(\mathrm{~s}, 3 \mathrm{H}, \mathrm{CH}_{3}\right), 2.49\left(\mathrm{~s}, 6 \mathrm{H}, \mathrm{CH}_{3}\right), 2.36$ (q, $\left.J=7.6 \mathrm{~Hz}, 4 \mathrm{H}, \mathrm{CH}_{2}\right), 1.40\left(\mathrm{~s}, 6 \mathrm{H}, \mathrm{CH}_{3}\right), 0.99\left(\mathrm{t}, J=7.5 \mathrm{~Hz}, 6 \mathrm{H}, \mathrm{CH}_{2} \mathrm{CH}_{3}\right)$.

${ }^{13} \mathrm{C}$ NMR (126 MHz, acetone-d6) $\delta 192.51$ (Ar), 154.43 (Ar), 154.29 (Ar), 151.70 (Ar), 141.58 (Ar), 139.14 (Ar), 134.59 (Ar), 133.77 (Ar), 133.57 (Ar), 133.22 (Ar), 133.15 (Ar), 131.47 (Ar), 129.00 (Ar), 128.73 (Ar), 109.60 (C-sp), 103.89 (C-sp), $39.22\left(\mathrm{CH}_{3}\right), 37.55\left(\mathrm{CH}_{3}\right), 32.13\left(\mathrm{CH}_{3}\right), 28.58\left(\mathrm{CH}_{3}\right)$, $17.49\left(\mathrm{CH}_{3}\right), 14.95\left(\mathrm{CH}_{3}\right), 12.61\left(\mathrm{CH}_{2}\right), 12.08\left(\mathrm{CH}_{3}\right)$.

${ }^{11} \mathrm{~B}$ NMR $\left(128 \mathrm{MHz}\right.$, acetone- $\left.d_{6}\right) \delta 0.77$.

ESI-MS $\left(\mathrm{CH}_{3} \mathrm{CN}\right.$, pos. mode) for $\mathrm{C}_{34} \mathrm{H}_{39} \mathrm{AuBF}_{2} \mathrm{~N}_{6} \mathrm{O}_{2}$ : exp. 809.2877 (calc. 809.2867).

Elemental analysis for $\mathrm{C}_{34} \mathrm{H}_{44} \mathrm{AuBF}_{2} \mathrm{~N}_{6} \mathrm{O}_{5}\left(\mathbf{4 d} \cdot 4 \mathrm{H}_{2} \mathrm{O}\right.$ ): exp. C 46.81\%, H 4.85\%, N $9.58 \%$ (calc. C $46.38 \%, \mathrm{H} 5.27 \%, \mathrm{~N} 9.54 \%)$

X-ray diffraction analysis. Complex 3a, 4b and 4d were allowed to form crystals by slow evaporation of acetonitrile at room temperature. The crystals were analysed at the UK National Crystallography Service in Southampton. A suitable colourless irregular-shaped crystal of 3a $\left(0.260 \times 0.110 \times 0.060 \mathrm{~mm}^{3}\right)$ and a colourless plate-shaped crystal of $\mathbf{4 b}\left(0.150 \times 0.080 \times 0.020 \mathrm{~mm}^{3}\right)$ were selected and mounted on a MITIGEN holder in perfluoroether oil on a Rigaku 007HF diffractometer equipped with Varimax confocal mirrors and an AFC11 goniometer and HyPix $6000 \mathrm{HE}$ detector. The crystal was kept at a steady $T=100.01(10) \mathrm{K}$ during data collection. In the case of complex 4d, several orange plate-shaped crystals with approximate dimensions $0.040 \times 0.025 \times 0.015 \mathrm{~mm}^{3}$ were mounted on a glass fibre in perfluoroether oil. Data were collected at 
the Diamond beamline I19 diffractometer equipped with a Helix cryostat low-temperature device operating at $\mathrm{T}=30(2) \mathrm{K}$. The data was collected using 360 degree phi scans at 5\%, and crystal 1 also using a 45 degree phi scan at $2 \%$. Since the first crystal was affected by radiation damage, data sets for several crystals were collected, and had to be scaled accordingly. Data were measured using monochromatic $\operatorname{Si}(111)$ radiation $(\lambda=0.6889 \AA)$. The maximum resolution that was achieved was $\Theta=23.905^{\circ}(0.85 \AA)$. The structures of $\mathbf{3 a}$ and $\mathbf{4} \mathbf{b}$ were solved with the ShelXD [39] structure solution program using the Dual Space solution method and by using Olex2 [40] as the graphical interface. Each model was refined with version 2014/7 of ShelXL [41] using Least Squares minimisation. The structure of $\mathbf{4 d}$ was solved and the space group $P 2{ }_{1} / c$ (\# 14) determined by the ShelXT (Sheldrick, 2015) structure solution program [41] using Intrinsic Phasing and by using Olex2 (Dolomanov et al., 2009) as the graphical interface [40]. The model was refined by Least Squares using version 2014/7 of ShelXL (Sheldrick, 2015) [42]. All non-hydrogen atoms were refined anisotropically. Hydrogen atom positions were calculated geometrically and refined using the riding model. There was a large volume $\left(1094 \AA^{3}\right)$ for solvents. No obvious solvents were identified, and the structure was SQUEEZEd. CCDC codes are 1916760, 1913564 and 1940532.

${ }^{1} \mathrm{H}$ NMR stability studies. ${ }^{1} \mathrm{H}$ NMR were recorded in a mixture DMSO- $d_{6}: \mathrm{D}_{2} \mathrm{O}(80: 20)$, with TMS as an internal reference, on a Bruker Avance 400 NMR spectrometer (Bruker Daltonics, Bremen, Germany). The gold complexes 3a-d and 4a-c $(6 \mathrm{mmol})$ have been dissolved in the solvent mixture chosen with or without an equimolar amount of NAC. ${ }^{1} \mathrm{H}$ NMR spectra were recorded at time 0 and after $24 \mathrm{~h}$ with each of the sample freshly prepared before the first run.

Stability studies by UV-visible spectroscopy. UV-visible absorption spectra to investigate the stability of compound $\mathbf{4 d}$ in solution were recorded on a Cary $60 \mathrm{UV}-\mathrm{Vis}$ spectrometer (Agilent Technologies, Santa Clara, USA). A stock solution of $\mathbf{4 d}(3 \mathrm{mM})$ was prepared in DMSO. An aliquot was diluted to $40.6 \mu \mathrm{M}$ either in $1 \times \mathrm{PBS}(\mathrm{pH} 7.4)$ or deionized water, and the UV-vis spectra acquired 
at room temperature over $24 \mathrm{~h}$ at different intervals (every 15 min during the first hour and every hour for the remaining $23 \mathrm{~h}$ ).

Quantum yield determination. Emission spectra were recorded on a Cary Eclipse fluorescence spectrophotometer (Agilent Technologies, Santa Clara, USA). Compound 4d was dissolved in degassed DMSO to a concentration corresponding to UV-vis absorbance 0.8 A.U. $\left(\lambda_{(\max )}=523-535\right.$ $\mathrm{nm} ; 25^{\circ} \mathrm{C}$ ). The solution was transferred to a fluorescence spectrophotometer and an emission spectrum was recorded (excitation wavelength $595 \mathrm{~nm}$ ). The quantum yield of complex 4 d was calculated by comparison to a reference standard (Rhodamine 6G in ethanol, $\varphi=94 \%$ ).

FRET DNA melting assay. Fluorescence resonance energy transfer (FRET) experiments were run on an Applied Biosystems ${ }^{\circledR}$ QuantumStudio 5 Real-Time PCR thermocycler (Thermo Fisher Scientific, Waltham, USA) equipped with a FAM filter $\left(\lambda_{\mathrm{ex}}=492 \mathrm{~nm} ; \lambda_{\mathrm{em}}=516 \mathrm{~nm}\right)$. The thermocycler was set to perform a stepwise increase of $0.3{ }^{\circ} \mathrm{C}$ every $30 \mathrm{~s}$, from $25{ }^{\circ} \mathrm{C}$ to $95{ }^{\circ} \mathrm{C}$, and measurements were acquired after each step.

All the oligonucleotides were purchased from Eurogentec (Belgium) in HPLC purity grade. The FRET probes used were FAM (6-carboxyfluorescein) and TAMRA (6-carboxytetramethylrhodamine). The lyophilized fluorolabelled hTelo (21-mer), d[GGG(TTAGGG) 3 , $C$ KIT1, d[GGGAGGGCGCTGGGAGGAGGG] and hTERT, d[GGGGGCTGGGCCGGGGACCCGGGAGGGGTCGGGACGGGGCGGGG] oligonucleotides were firstly diluted in deionized water to obtain $100 \mu \mathrm{M}$ stock solutions. Stock solutions were diluted to a concentration of $400 \mathrm{nM}$ in potassium cacodylate buffer $(54 \mathrm{mM}, \mathrm{pH} 7.4)$, and then annealed to form G-quadruplex (G4) structures by heating to $95^{\circ} \mathrm{C}$ for $5 \mathrm{~min}$, followed by cooling to room temperature overnight.

Experiments were carried out in a 96-well plate with a total volume of $30 \mu \mathrm{L}$. The final concentration of the G4-oligonucleotide was set to $200 \mathrm{nM}$ in potassium cacodylate buffer (54 mM, pH 7.4). Stock 
solutions of the gold compounds 3a-d and 4a-c in DMSO $(1 \mathrm{mM})$ were freshly prepared prior to the experiments. The stock solutions were further diluted to a final concentration of $2 \mu \mathrm{M}$ (with a total percentage of DMSO of approx. $0.1 \%$ ) in potassium cacodylate buffer (54 mM, pH 7.4) to achieve G4 : gold compound stoichiometry of $1: 5$.

To perform the competition assays with duplex DNA, a $100 \mu \mathrm{M}$ stock solution in MilliQ water of annealed double-stranded DNA (dsDNA), d[GGGTTACTACGAACTGG]/d[CCAGTTCGTAGTAACCC], was purchased from Eurogentec (Belgium) in HPLC purity grade. This solution was further diluted to $40 \mu \mathrm{M}$ in $54 \mathrm{mM}$ potassium cacodylate buffer ( $\mathrm{pH}$ 7.4). Stock solutions of the gold compounds 3a-d and 4a-c were diluted to 4 $\mu \mathrm{M}$ (with a total percentage of DMSO of approx. $0.1 \%$ ) in potassium cacodylate buffer $(54 \mathrm{mM}, \mathrm{pH}$ 7.4). The final concentration of the G4-oligonucleotide was $200 \mathrm{nM}$, with a stoichiometry of $1: 5: 50$ of G4 : gold compound : dsDNA. Experiments were carried out in a 96-well plate with a total volume of $30 \mu \mathrm{L}$. To compare different sets of data, FAM emission was normalised ( 0 to 1$)$. $\mathrm{T}_{\mathrm{m}}$ is defined as the temperature at which the normalised emission is 0.5 and $\Delta \mathrm{T}_{\mathrm{m}}$ is defined as the difference of $\mathrm{T}_{\mathrm{m}}$ between treated samples and untreated controls. Independent experiments were run in triplicates.

Circular dichroism. Circular Dichroism (CD) spectra were recorded on a ChiraScan (AppliedPhotophysics, Surrey, UK) using the following parameters: range 210-400 nm, bandwidth $1 \mathrm{~nm}$, step $1 \mathrm{~nm}$, accumulation 3 , temperature $25^{\circ} \mathrm{C}$. Stock solutions of unlabelled 21-mer hTelo, d[GGG(TTAGGG) 3 ] and $C$-KIT1, d[GGGAGGGCGCTGGGAGGAGGG] (each $100 \mu \mathrm{M}$ ), were prepared by dissolving the lyophilised sample in deionized water. Samples were further diluted in a Tris- $\mathrm{HCl} / \mathrm{KCl}$ buffer $(10 / 50 \mathrm{mM}, \mathrm{pH} 7.4)$ to obtain a final concentration of $4 \mu \mathrm{M}$. Stock solutions of AuTMX2, 3a and 3c were prepared as described in the previous section and further diluted in buffer to achieve a G4 : gold compound stoichiometry of $1: 5$. 
Cell lines and culture maintenance. The human cancer cell lines corresponding to breast carcinoma (MCF-7), ovarian adenocarcinoma (SKOV-3) and skin malignant melanoma (A375) were obtained from the ATCC. The SKOV-3 and A375 cells were cultured in Dulbecco`s Modified Eagle Medium (DMEM, 4,5 g/L glucose, Corning), supplemented with 10\% fetal bovine serum (One-Shot FBS, EUapproved South American Origin, Thermo Fisher Scientific) and 1\% penicillin/streptomycin (Gibco). MCF-7 cells were grown in Roswell Park Memorial Institute medium (RPMI, L-glutamine, Corning), supplemented with 10\% fetal bovine serum (One Shot FBS, EU-approved South American Origin, Thermo Fisher Scientific) and 1\% penicillin/streptomycin (Gibco). All cell lines were cultured at 37 ${ }^{\circ} \mathrm{C}$, in a humidified atmosphere of $5 \% \mathrm{CO}_{2}$ and passage diluted upon reaching confluence.

Antiproliferative assays. To evaluate the inhibition of cell growth by the gold complexes, cells were seeded in 96-well tissue culture-treated plates (Corning) at 8'000 cells/well in $200 \mu \mathrm{L}$ full medium. Working solutions of the gold compounds $3 \mathbf{a}-\mathbf{c}, \mathbf{4 a}-\mathbf{d}$ were prepared in the required concentration by diluting freshly prepared stock solutions $\left(10^{-2} \mathrm{M}\right.$ in DMSO) in complete medium. The stock solutions were protected from light in order to avoid potential light-induced degradation. Cells were allowed to adhere for $24 \mathrm{~h}$. Then, the medium was refreshed and cells were incubated for $72 \mathrm{~h}$ in $200 \mu \mathrm{L}$ complete medium containing different concentrations of the gold compounds. The antiproliferative effects of complexes were evaluated using the 3-(4,5-dimethylthiazol-2-yl)-2,5-diphenyltetrazolium bromide (MTT) assay. Following $72 \mathrm{~h}$ exposure, the medium was removed and 3-(4,5dimethylthiazol-2-yl)-2,5-diphenyltetrazolium bromide (MTT, Fluorochem) in 10× PBS (Corning) was added to the cells, at a final concentration of $0.3 \mathrm{mg} / \mathrm{mL}$. After $3-4 \mathrm{~h}$ incubation at $37{ }^{\circ} \mathrm{C}$ and $5 \%$ $\mathrm{CO}_{2}$, the supernatant was discarded and the formazan crystals were dissolved with DMSO. The optical density was quantified in quadruplicates for each experiment at $550 \mathrm{~nm}$ using a multi-well plate reader (VICTOR X, Perking Elmer).

Complex 4d was evaluated using the CellTiter-Blue ${ }^{\circledR}$ assay due to the interference with the MTT absorbance. After $72 \mathrm{~h}$ incubation with the compound, CellTiter-Blue ${ }^{\circledR}$ reagent $(20 \mu \mathrm{L} /$ well $)$ was 
added to the assay plate, shaken for $10 \mathrm{~s}$ and incubated for $4 \mathrm{~h}$ at $37^{\circ} \mathrm{C}$ and $5 \% \mathrm{CO}_{2}$. Fluorescence intensity $\left(531_{\mathrm{Ex}} / 595_{\mathrm{Em}} \mathrm{nm}\right)$ from each well was measured in quadruplicates for each experiment using a multi-well plate reader (VICTOR X5, Perking Elmer).

The $\mathrm{EC}_{50}$ value for each compound in both assays was calculated as the concentration showing $50 \%$ decrease in cell growth, when compared to controls, using a nonlinear fitting. In the case of compound 4d fluorescence intensity was corrected for interference of the BODIPY-alkyne. Data is presented as mean \pm standard deviation of at least three independent experiments.

Fluorescence microscopy. Round glass coverslips (Ø 13mm, VWR) sterilized by UV-light were inserted in 24-well tissue culture-treated plates (Corning). A375 cells were seeded at a concentration of $1200^{\prime} 000$ cells $/ \mathrm{mL}$ and incubated at $37^{\circ} \mathrm{C}$ under humidified atmosphere with $5 \% \mathrm{CO}_{2}$ for $24 \mathrm{~h}$. The medium was discarded and fresh medium containing $15 \mu \mathrm{M}$ of complex $\mathbf{4 d}$ was added. Following 2 h incubation at $37^{\circ} \mathrm{C}$, the glass coverslips were removed from the wells, washed four times with $1 \times$ PBS (Corning) and fixed with $4 \%$ formaldehyde (Alfa-Aesar) for $20 \mathrm{~min}$ at room temperature. The coverslips were then washed three times with $1 \times$ PBS and incubated for $1 \mathrm{~min}$ at room temperature with 4',6-diamidino-2-phenylindole dihydrochloride (DAPI, $40 \mu \mathrm{L}$ of $1 \mu \mathrm{g} / \mathrm{mL}$, SigmaAldrich/MERCK). After washing the coverslips three times with $1 \times$ PBS they were mounted on glass microscope slides (VWR) using Mowiol ${ }^{\circledR}$ 4-88 (Sigma-Aldrich). Fluorescence images were obtained using a Zeiss Axio Vert.A1 epifluorescence microscope and analysed using ImageJ software [43]. 


\section{RESULTS AND DISCUSSION}

\subsection{Synthesis and characterisation}

Initially, we synthesized a family of cationic gold(I) bis-NHC complexes related to the lead compound AuTMX2. Specifically, the N1 position of theobromine was substituted with ethyl (a), (2cyclohexyl)ethyl (b), benzyl (c) and 4-(trifluoromethyl)benzyl (d) moieties (Scheme 1).

\section{Scheme 1.}

The synthesis of the functionalised ligands (1a-d) was confirmed by ${ }^{1} \mathrm{H}$ NMR by the disappearance of the broad peak of the N1-proton and by the appearance of the signals related to the newly introduced groups (Fig. S1-4). Next, the N1-substituted ligands were methylated in the N9-position using Merweein's salt. This is a crucial step since only the positively charged tetraalkylxanthinium precursor facilitates carbene formation and subsequent complexation to $\mathrm{Au}(\mathrm{I})$ [37]. The xanthinium ligands (2a-d) were isolated and the methylation was confirmed by ${ }^{1} \mathrm{H},{ }^{13} \mathrm{C}$ NMR and elemental analysis. As evident from the ${ }^{1} \mathrm{H}$ NMR spectra (Fig. S9-12), the methylation in N9-position resulted in the appearance of a singlet with nearly identical chemical shift compared to the N7-methyl signal, together integrating for 6 protons $(4.08 \mathrm{ppm})$, whereas the $\mathrm{C} 8$ proton experienced a downfield shift to $8.48 \mathrm{ppm}$. Furthermore, a new methyl-specific carbon signal appeared at $37.5 \mathrm{ppm}$ (Fig. S13-S16).

The final four gold(I) complexes (3a-d) were obtained via a transmetalation reaction (Scheme 1) [37]. The NHCs were formed in situ in the presence of $\mathrm{Ag}_{2} \mathrm{O}$ under inert atmosphere and were stabilized by $\mathrm{Ag}$-complexation. Addition of $\mathrm{Au}(\mathrm{tht}) \mathrm{Cl}$ induced transmetallation and silver chloride precipitated from solution. The gold(I) bis-NHC complexes were obtained in moderate yields. Deprotonation of the C8 position was confirmed by ${ }^{1} \mathrm{H}$ NMR. Upon complexation, the methyl peaks at 4.08 ppm split more clearly into two distinct signals for $\mathbf{3 a}, \mathbf{3 c}$ and $\mathbf{3 d}$. Moreover, in the ${ }^{13} \mathrm{C}$ NMR spectra these three complexes also experienced a ca. 2 ppm downfield shift of the N9-methyl signal (see Fig. S17,19, 20). The ${ }^{1} \mathrm{H}$ NMR spectrum of $\mathbf{3 b}$ showed the presence of two simultaneously 
existing coordination states, as indicated by the appearance of two sets of methyl signals with constant ratios, but only one set of signals corresponding to the (2-cyclohexy)ethyl moiety, probably corresponding to rotamers (Figures S18 and S22).

The second series of compounds included the mixed NHC-alkynyl gold(I) complexes (4a-d), which were readily obtained from reacting the neutral mono-carbene complex AuTMXI with the respective deprotonated alkyne in the presence of a base (Scheme 2) [20]. Interestingly, complex 4d features a boron-dipyrromethene (BODIPY)-based alkynyl ligand with luminescence properties which can allow the compound's visualization in cells by fluorescence microscopy.

Scheme 2.

The successful synthesis of the complexes 4a-d was confirmed by ${ }^{1} \mathrm{H}$ and ${ }^{13} \mathrm{C}$ NMR (Fig. S25-S33), elemental analysis and in some cases by ESI-MS (Fig. S36, S37). The appearance of a signal at $108 \mathrm{ppm}$ in the ${ }^{13} \mathrm{C}$ NMR spectra is characteristic for the coordinated alkynyl. Single crystals of 3a, $\mathbf{4 b}$ and $4 \mathbf{d}$ were obtained by slow evaporation of acetonitrile. The structures are shown in Figure 2 while the relevant crystal data are reported in the supplementary material (see Tables S2-S26).

Figure 2.

The three compounds show a linear geometry with the angle $\mathrm{C}-\mathrm{Au}-\mathrm{C}$ being $178.54^{\circ}, 179.10^{\circ}$ and $176.79^{\circ}$ for $\mathbf{3 a}, \mathbf{4 b}$ and $\mathbf{4 d}$, respectively. A monoclinic crystal system was resolved for $\mathbf{3 a}$, where the complex forms intramolecular hydrogen bonds with a water molecule (see Figure S54), whereas 4b is characterised by a triclinic crystal system with three molecules are staggered upon each other. In the latter, the distance between each molecule is $3.610 \AA$, which is compatible with the occurrence of $\pi-\pi$ interactions between the complexes. Moreover, aurophilic interactions [44] among the three $\mathbf{4 b}$ complexes were also found, as evidenced by the gold-gold distance of $3.286 \AA$. Noteworthy, the triclinic crystal system resolved for $\mathbf{4 d}$ shows the existence of two distinct arrangements of the 
molecule within the asymmetric unit (see Figure 2C, Figure S55 and tables S18-S26 for more information). In each complex bond distances between the gold(I) centre and either the C8 of TBM and/or the alkynyl carbon are $2.0 \AA$, similarly to those reported for other TMX-based gold(I) organometallics [37]. Noteworthy, the crystal of 3a confirms that, differently from what previously reported for the N9 substitution, the N1-modified complexes retains their planarity.

Next, the quantum yield of the gold compound $\mathbf{4 d}$ and the BODIPY-alkyne ligand precursor were determined in aqueous solution (see Table 1 and experimental section for details). Complexation of the fluorophore to the gold(I) NHC complex via the alkynyl bridge did not quench the fluorescence of the resulting metal complex, whose quantum yield was found to be $74 \%$. Moreover, gold(I) complexation did not change the absorption and emission wavelengths of the BODIPY fluorophore.

Table 1.

\subsection{Stability studies}

The stability of the gold(I) bis-NHCs and mixed NHC-alkynyl complexes was studied over $24 \mathrm{~h}$ in a mixture DMSO- $d_{6}: \mathrm{D}_{2} \mathrm{O}(80: 20)$ by ${ }^{1} \mathrm{H}$ NMR (Figures S38-S44). The compounds were largely stable, except for $\mathbf{4 a}$, for which a new set of peaks appeared in the region from 2.5 to $4.0 \mathrm{ppm}$ after 24 h, most likely due to the displacement of the alkynyl moiety by DMSO (see Figure S42).

In order to characterize the reactivity of the gold(I) complexes with model nucleophiles, the compounds 3a-d and $\mathbf{4 b - c}$ were exposed to an equimolar amount of $\mathrm{N}$-acetylcysteine (NAC) and

analysed by ${ }^{1} \mathrm{H}$ NMR spectroscopy. The stability of NAC alone was also followed over time by ${ }^{1} \mathrm{H}$ NMR. NAC underwent auto-oxidation to cystine to an extent of ca. $30 \%$ after $24 \mathrm{~h}$ as observed by the formation of a new set of signals at 4.45, 3.09 and 1.87 ppm (Fig. S45). All samples showed a similar cystine : cysteine ratio in the presence or absence of the gold(I) complexes.

The results obtained here show that the compounds are largely stable (Figures S46-51). Compounds $\mathbf{3 b}, \mathbf{3 c}$ and $\mathbf{4 b}$ displayed some changes in their ${ }^{1} \mathrm{H}$ NMR spectra over $24 \mathrm{~h}$ (Fig S47, S48 
and S50). In detail, for $\mathbf{3 b}$ over 24 hours the signals related to the methyl protons are shifted to 3.99 and $3.66 \mathrm{ppm}$ while the third one disappears probably hidden below the solvent signal (Fig. S47). Moreover, a new small signal appears at 2.97 which can be attirbuted to the Au-bound cysteine. The peaks at 5.05 and $3.71 \mathrm{ppm}$ for $\mathbf{3 c}$ are split over time while two more signals for the methyl groups appear at 3.99 and 3.96 respectively. Analysis of the integrals suggests the existence of a 1:2 ratio between the original compound and a reaction product that may tentatively be attributed to a cysteine adduct (Fig. S48). The latter is also confirmed by the appearance of new peaks at 3.05 and $2.97 \mathrm{ppm}$ whose signals are related to Au-bound cysteine. Similarly, as detailed in Fig. S50, $\mathbf{4 b}$ experiences a splitting of the methyl signals at 4.10, 3.97 and $3.20 \mathrm{ppm}$, while a new signal appears for the Aubound cysteine $(2.95 \mathrm{ppm})$ likely due to the protons close to the sulphur atom. Two additional peaks are found in the aromatic region, respectively at 8.54 and $7.45 \mathrm{ppm}$, which clearly show the displacement of the aromatic-alkynyl moiety by NAC, in a $\mathbf{4 b}:$ cysteine-adduct $=70: 30$. Overall, compounds 3a, 3d and $\mathbf{4 c}$ are inert with respect to ligand exchange reactions with NAC over $24 \mathrm{~h}$, while $\mathbf{3 b}, \mathbf{3} \mathbf{c}$ and $\mathbf{4 b}$ are prone to react with NAC, leading to the probable formation of sulfur-adducts. Due to the scarce solubility in DMSO, the stability of the BODIPY-based complex $\mathbf{4 d}$ could not be evaluated by NMR, and thus, UV-vis stability studies were conducted. The experiments were carried out over $24 \mathrm{~h}$ in $1 \times$ Phosphate Buffered Saline $(\mathrm{PBS}, \mathrm{pH}=7.4)$ and deionized water, with data collection each $15 \mathrm{~min}$ for the first hour and then each hour for the remaining 23 hours. The UV-vis spectra of 4d are characterised by two intense bands at 533 and $293 \mathrm{~nm}$ and a shoulder in between at $380 \mathrm{~nm}$ (black solid line in Fig. S52). Over $24 \mathrm{~h}$ these bands experienced hypochromic effects and markedly decreased in PBS buffer (Fig. S52). A similar hypochromism is evident in deionised water, where $4 \mathbf{d}$ is more stable than in PBS. In both solvents, the intensity of the absorption is reduced by ca. 30-40\% after $6 \mathrm{~h}$. These hypochromic effects may be attributable to the hydrolysis of the B-F moiety in the BODIPY scaffold leading to precipitation. In fact, a similar and even more prominent trend can be observed for the free BODIPY ligand (Fig. S53). 


\subsection{G4-stabilisation studies}

The DNA G-quadruplex (G4) stabilising properties of the newly synthesised $\mathrm{Au}(\mathrm{I}) \mathrm{NHC}$ organometallics were assessed by means of fluorescence resonance energy transfer (FRET) DNA melting assays [45]. Earlier studies with cationic gold(I) bis-NHC complexes achieved after N9 substitution on the lead compound AuTMX 2 focused on telomeric G4s [37]. Here, we extended these studies to two oncogenic promoter G4 structures, namely, $C$-KITl and $h T E R T$. The former is an oncogenic receptor tyrosine kinase involved in proliferation [46], and the latter is the catalytic subunit of the human telomerase, which is involved in cancer immortality [47].

The gold compounds 3a-d and 4a-c were reacted with each G4 in a 5:1 stoichiometry as detailed in the experimental section. Table $\mathrm{S} 1$ shows the $\Delta \mathrm{T}_{\mathrm{m}}$ for each compound in comparison to the previously reported complexes, the cationic bis-carbene derivative AuTMX 2 and the mono-carbene AuTMXI.

Interestingly, AuTMX 2 is most potently stabilising hTelo $\left(\Delta \mathrm{T}_{\mathrm{m}}=13.1 \pm 1.8{ }^{\circ} \mathrm{C}\right)$ in comparison to the gene promoter G4s of $C$-KIT1 and hTERT (Table S1). In contrast, AuTMXI can similarly stabilize $C$-KITI $\left(\Delta \mathrm{T}_{\mathrm{m}}=4.0 \pm 0.1{ }^{\circ} \mathrm{C}\right)$ and hTelo $\left(\Delta \mathrm{T}_{\mathrm{m}}=3.2 \pm 0.6^{\circ} \mathrm{C}\right)$, while it is practically inactive towards $h T E R T\left(\Delta \mathrm{T}_{\mathrm{m}}=1.54 \pm 0.15^{\circ} \mathrm{C}\right)$. Concerning the newly synthesized compounds, extending the N1-methyl in 3a-d also led to a marked reduction of their G4 stabilizing potency, similarly to N9 modifications [37]. The best stabilizer of this series is complex 3a, showing a similar trend compared to AuTMX2, while 3c was able to slightly stabilize the hTelo DNA sequence (Figures 3A and 4B). It may be noted that 3a differs from AuTMX2 only by ethyl to methyl substituents at the N1-position.

The mixed Au(I) NHC-alkynyl complexes 4a-c did not show any stabilising effects on the G4 structures. Despite the fact that the flat aromatic surface of these mixed NHC-alkynyl complexes could have allowed favourable $\pi-\pi$ interactions with the G4s, it can be hypothesized that the steric demand of 4a-c does not allow the compounds' alignment with the G4-quartet. The intrinsic fluorescence of $\mathbf{4 d}$ did not allow its screening by FRET.

In addition to their stabilizing potency, the selectivity of the compounds' for G4s and duplex DNA binding was investigated for AuTMX2, 3a and 3c with hTelo and $C$-KIT1 in the presence of a 
50-fold excess of dsDNA. The three organometallics showed a clear selective interaction towards both hTelo and C-KIT1 over dsDNA, as their stabilising properties were unperturbed when challenged with the excess of dsDNA (Fig. 3C, D). In the previously reported study, N9-substituted $\mathrm{Au}(\mathrm{I})$ bis-NHC complexes showed increased affinity towards dsDNA [37]. Consequently, although the N1-modified $\mathrm{Au}(\mathrm{I})$ bis-NHC complexes are less potent in stabilizing the G4 structures compared to N9-modified analogues, as assessed by $\Delta \mathrm{T}_{\mathrm{m}}$, the compounds retain their high selectivity for G4 binding.

Figure 3.

Furthermore, circular dichroism (CD) spectra of hTelo and C-KITI in the presence of 5 equiv. of the G4 stabilising compounds AuTMX2, 3a and 3c (only hTelo) were recorded to assess if any changes of the G4-folding would occur upon interaction of the polynucleotide with the gold(I) NHC complexes. In the UV-vis range, the G4-DNA features two $\pi-\pi^{*}$ transitions (at ca. 280 and $248 \mathrm{~nm}$ ). However, within a G-quadruplex, the tetrads are rotated with respect to each other giving rise to a chiral exciton coupling between transition dipole moments located in near-neighbour guanines: the final G4-DNA is therefore, CD active [48]. In detail, the CD spectrum of hTelo in our experimental conditions is characterised by an intense positive band at $290 \mathrm{~nm}$ with a shoulder at $270 \mathrm{~nm}$ and by a negative absorption at $240 \mathrm{~nm}$ (black solid line in Fig. 4A). This spectrum is typical of a hybrid (3+1) G4-conformation [49]. Conversely, in the same conditions, C-KIT1 folds in a parallel conformation with a positive band at about $263 \mathrm{~nm}$ and a negative centred at $240 \mathrm{~nm}$ (black solid line in Fig. 4B).

Figure 4.

Interestingly, when a $4 \mu \mathrm{M}$ solution of hTelo in Tris- $\mathrm{HCl} / \mathrm{KCl}(10 \mathrm{mM} / 50 \mathrm{mM}, \mathrm{pH} 7.4)$ is challenged with 5 equiv. of AuTMX 2 , 3a or 3c the positive band at $290 \mathrm{~nm}$ increases, as a result of the interaction 
of the three compounds with the G4-DNA as previously reported for other G4-binders (Fig. 4A) [5052]. The addition of AuTMX2 also causes a reduction of the shoulder at $270 \mathrm{~nm}$ which might suggest a slight structural change of the G4-DNA as previously observed with a nickel(II) complex [52]. The other two complexes, $\mathbf{3 a}$ and $\mathbf{3 c}$ do not show a similar decrease of the band located at $270 \mathrm{~nm}$, while 3c even induces an opposite modification of the signal. On the contrary, with respect to $C$-KITl, the CD analysis reported in Fig. 4B clearly shows that the interaction of the two complexes with the G4DNA, proven by the FRET DNA melting assays, occurs without affecting the secondary structure of the polynucleotide, as reported in literature for some nickel(II) and zinc(II) Schiff-base complexes [53].

\subsection{Antiproliferative assays}

The cytotoxicity of the compounds 3a-c and 4a-d was evaluated in human melanoma (A375), ovarian (SKOV-3) and breast (MCF-7) cancer cell lines in comparison to AuTMX2 (Table 2). It may be noted that AuTMX2 is completely inactive in these cancer cells, and AuTMXI also showed scarce cytotoxicity in all the tested cell lines.

Table 2.

Similarly to AuTMX2, the cationic Au(I) bis-NHC complex 3a, featuring an ethyl substituent at the N1-position, is inactive in all tested cell lines. Interestingly, when a bulkier modification is introduced, as in $\mathbf{3 b}$ and $\mathbf{3 c}$, a considerable increase in the cytotoxicity is observed towards all cancer cells, irrespective of being saturated (3b) or aromatic (3c). The tested $\mathrm{Au}(\mathrm{I})$ mixed NHC-alkynyl complexes, although scarcely active in all cell lines, showed a distinct activity profile with $\mathbf{4 a - c}$ moderately cytotoxic in the MCF-7 breast cancer cell line. Especially the 3-pyridine derivative 4c was slightly more active ( $\mathrm{IC}_{50}$ ca. $16 \mu \mathrm{M}$ ) than the other derivatives of this family. Finally, $\mathbf{4 d}$ has no cytotoxic effect against any of the three cancer cell lines. 


\subsection{Fluorescence microscopy}

Compound 4d bears a fluorescent BODIPY-moiety, but showed no cytotoxicity against the investigated cancer cell lines. In order to investigate whether the reduced cytotoxicity might correlate with a reduced cellular accumulation, we studied its uptake by fluorescence microscopy. Compound $4 \mathbf{d}(15 \mu \mathrm{M})$ was incubated with A375 cells for $2 \mathrm{~h}$ at $37^{\circ} \mathrm{C}$. The compound was found to be efficiently taken up by the cancer cells, and to accumulate in the cytoplasm (Figure 5), while cell nuclei did not show any signal corresponding to the BODIPY fluorophore. Thus, the low cytotoxicity of $\mathbf{4 d}$ might rather be related to a reduced stability as indicated by UV-vis experiments than by a reduced cellular accumulation. Since the absorption and emission wavelengths of $\mathbf{4 d}$ and the BODIPY-alkyne are very similar, it is challenging to judge whether the fluorescence is caused by intact $\mathbf{4 d}$ or free BODIPY ligand.

Figure 5.

\section{CONCLUSIONS}

The present study comparatively evaluated two novel families of gold(I) bis-N-heterocyclic carbene and mixed NHC-alkynyl organometallics with respect to their stability in aqueous environment, Gquadruplex (G4) stabilising effect and cytotoxicity. To the best of our knowledge this is one of the few reports in the literature on the interactions of metal NHCs featuring xhantine type of ligands with G4 DNA structures[31]. The representatives of both families form linear and highly planar gold(I) organometallic complexes, which were reasonably stable in aqueous solution. Upon interaction with NAC, complexes $\mathbf{3 b}, \mathbf{3 c}$ and $\mathbf{4 b}$ showed some ligand exchange reaction, involving substitution of the alkynyl ligand in the latter case. The cationic gold(I) N1-modified bis-NHCs exhibited mild G4 stabilisation properties, while the mixed NHC-alkynyl family was devoid of any G4 stabilising effects. Although less potent than the lead compound AuTMX2, the cationic gold(I) bis-NHC 
derivatives retained their selectivity for the G4 structures even in the presence of excess doublestranded DNA. While the complexes 3b-c are moderately cytotoxic in all cancer cell lines, the gold(I) mixed NHC-alkynyl complexes, though being less active, were selectively cytotoxic against the MCF-7 breast cancer cell line. Finally, a non-cytotoxic fluorescent gold(I) derivative (4d) was shown to efficiently distribute into the cytoplasm of intact cells after $2 \mathrm{~h}$ incubation with melanoma cells, but did not enter the nuclei. Overall, these investigations provide further insight into the distinct biological activity of gold(I) organometallics and underline their promise for tuneable biological applications by appropriate chemical modifications.

\author{
Abbreviations \\ $\operatorname{AuTMX}_{2} \quad\left[\operatorname{Au}(9-\text { methylcaffein-8-ylidene })_{2}\right]^{+}$ \\ AuTMXI [Au(9-methylcaffein-8-ylidene)I $]$ \\ BODIPY boron-dipyrromethene \\ C-KIT1 C-KIT1 oncogenic receptor tyrosine kinase \\ CD circular dichroism \\ DCM dichloromethane \\ DMF dimethylformamide \\ DMSO dimethylsulfoxide \\ dsDNA double-stranded DNA \\ ESI-MS electrospray ionization-mass spectrometry \\ FRET fluorescence resonance energy transfer \\ G4 G-quadruplex \\ hTelo G4 human telomeric G4 \\ hTERT human telomerase \\ MeCN acetonitrile \\ $\mathrm{MeOH} \quad$ methanol
}




$\begin{array}{ll}\text { N1-TBM } & \text { 1-alkyl-9methyltheobromin-8-ylidene } \\ \text { NAC } & \text { N-acetyl cysteine } \\ \text { NHC } & \text { N-heterocyclic carbene } \\ \text { NMR } & \text { nuclear magnetic resonance } \\ \text { PBS } & \text { phosphate buffered saline } \\ \text { tht } & \text { tetrahydrothiophene } \\ \text { TMX } & \text { tetramethylxanthine } \\ \text { Trx } & \text { thioredoxin reductase }\end{array}$

\section{Acknowledgements}

Cardiff University is acknowledged for funding. The authors are grateful to the Life Science Research Network Wales for providing a post-doctoral fellowship to S.M.M.. R.B. acknowledges funding from the European Union's Horizon 2020 research and innovation program under the Marie SkłodowskaCurie grant agreement $n^{\circ} 663830$.

\section{Author contributions}

S.M.M., A.C. and R.B. designed the study; S.M.M., R.B. and D.D. synthesized compounds; S.M.M and R.B. carried out the FRET DNA melting analysis; W.T.K. and S.J.C. performed X-ray data acquisition and analysis ; R.B., N.S. and L.L. performed stability studies and CD experiments; B.A. performed the antiproliferative in vitro essays and the fluorescence microscopy experiments. S.M.M., A.C. and R.B. wrote the manuscript, which was revised and approved by all authors.

\section{Conflict of interest}

The authors declare no conflict of interest. 


\section{References}

[1] S.P. Fricker, Medical uses of gold compounds: Past, present and future, Gold Bull. 29 (1996) 53-60. doi:10.1007/BF03215464.

[2] G.J. Higby, Gold in medicine: a review of its use in the West before 1900, Gold Bull. 15 (1982) 130-140. doi:10.1007/BF03214618.

[3] C.F. Shaw, Gold-Based Therapeutic Agents, Chem. Rev. 99 (1999) 2589-2600. doi:10.1021/cr980431o.

[4] S. Nobili, E. Mini, I. Landini, C. Gabbiani, A. Casini, L. Messori, Gold compounds as anticancer agents: chemistry, cellular pharmacology, and preclinical studies, Med. Res. Rev. 30 (2010) 550-580. doi:10.1002/med.20168.

[5] J. Hu, H. Zhang, M. Cao, L. Wang, S. Wu, B. Fang, Auranofin Enhances Ibrutinib's Anticancer Activity in EGFR-Mutant Lung Adenocarcinoma, Mol. Cancer Ther. 17 (2018) 2156. doi:10.1158/1535-7163.MCT-17-1173.

[6] C. Roder, M.J. Thomson, Auranofin: Repurposing an Old Drug for a Golden New Age, Drugs RD. 15 (2015) 13-20. doi:10.1007/s40268-015-0083-y.

[7] A. Jatoi, C. Radecki Breitkopf, N.R. Foster, M.S. Block, M. Grudem, A. Wahner Hendrickson, R.E. Carlson, B. Barrette, N. Karlin, A.P. Fields, A Mixed-Methods Feasibility Trial of Protein Kinase C Iota Inhibition with Auranofin in Asymptomatic Ovarian Cancer Patients, Oncology. 88 (2015) 208-213. doi:10.1159/000369257.

[8] S.M. Meier-Menches, C. Gerner, W. Berger, C.G. Hartinger, B.K. Keppler, Structure-activity relationships for ruthenium and osmium anticancer agents - towards clinical development, Chem. Soc. Rev. 47 (2018) 909-928. doi:10.1039/C7CS00332C.

[9] B. Bertrand, A. Casini, A golden future in medicinal inorganic chemistry: the promise of anticancer gold organometallic compounds, Dalton Trans. 43 (2014) 4209-4219. doi:10.1039/c3dt52524d.

[10] A. Casini, R.W.-Y. Sun, I. Ott, Medicinal Chemistry of Gold Anticancer Metallodrugs, in: A. Sigel, H. Sigel, E. Freisinger, R.K.O. Sigel (Eds.), Met.-Drugs Dev. Action Anticancer Agents, De Gruyter, Berlin, Boston, 2018: pp. 199218. doi:10.1515/9783110470734-013.

[11] A. Casini, R. Bonsignore, J. Oberkofler, Organometallic Gold-Based Anticancer Therapeutics, in: Ref. Module Chem. Mol. Sci. Chem. Eng., Elsevier, 2018: pp. 10.1016/B978-0-12-409547-2.14350-1. doi:10.1016/B978-0-12409547-2.14350-1.

[12] M. Mora, M.C. Gimeno, R. Visbal, Recent advances in gold-NHC complexes with biological properties, Chem. Soc. Rev. 48 (2019) 447-462. doi:10.1039/C8CS00570B.

[13] P.J. Barnard, M.V. Baker, S.J. Berners-Price, D.A. Day, Mitochondrial permeability transition induced by dinuclear gold(I)-carbene complexes: potential new antimitochondrial antitumour agents, J. Inorg. Biochem. 98 (2004) 1642-1647. doi:10.1016/j.jinorgbio.2004.05.011. 

linear $\mathrm{Au}(\mathrm{I}) \mathrm{N}$-heterocyclic carbene complexes: synthesis, structure and anti-mitochondrial activity, Dalton Trans. Camb. Engl. 2003. (2006) 3708-3715. doi:10.1039/b602560a.

[15] J.L. Hickey, R.A. Ruhayel, P.J. Barnard, M.V. Baker, S.J. Berners-Price, A. Filipovska, Mitochondria-Targeted Chemotherapeutics: The Rational Design of Gold(I) N-Heterocyclic Carbene Complexes That Are Selectively Toxic to Cancer Cells and Target Protein Selenols in Preference to Thiols, J. Am. Chem. Soc. 130 (2008) 12570-12571. doi:10.1021/ja804027j.

[16] C. Schmidt, B. Karge, R. Misgeld, A. Prokop, R. Franke, M. Brönstrup, I. Ott, Gold(I) NHC Complexes: Antiproliferative Activity, Cellular Uptake, Inhibition of Mammalian and Bacterial Thioredoxin Reductases, and GramPositive Directed Antibacterial Effects, Chem. - Eur. J. 23 (2017) 1869-1880. doi:10.1002/chem.201604512.

[17] R. Rubbiani, S. Can, I. Kitanovic, H. Alborzinia, M. Stefanopoulou, M. Kokoschka, S. Mönchgesang, W.S. Sheldrick, S. Wölfl, I. Ott, Comparative in vitro evaluation of N-heterocyclic carbene gold(I) complexes of the benzimidazolylidene type, J. Med. Chem. 54 (2011) 8646-8657. doi:10.1021/jm201220n.

[18] X. Cheng, P. Holenya, S. Can, H. Alborzinia, R. Rubbiani, I. Ott, S. Wölfl, A TrxR inhibiting gold(I) NHC complex induces apoptosis through ASK1-p38-MAPK signaling in pancreatic cancer cells, Mol. Cancer. 13 (2014) 221. doi:10.1186/1476-4598-13-221.

[19] P. Holenya, S. Can, R. Rubbiani, H. Alborzinia, A. Jünger, X. Cheng, I. Ott, S. Wölfl, Detailed analysis of proapoptotic signaling and metabolic adaptation triggered by a N-heterocyclic carbene-gold(I) complex, Metallomics. 6 (2014) 1591-1601. doi:10.1039/C4MT00075G.

[20] N. Estrada-Ortiz, F. Guarra, I.A.M. de Graaf, L. Marchetti, M.H. de Jager, G.M.M. Groothuis, C. Gabbiani, A. Casini, Anticancer Gold N-Heterocyclic Carbene Complexes: A Comparative in vitro and ex vivo Study, ChemMedChem. 12 (2017) 1429-1435. doi:10.1002/cmdc.201700316.

[21] Ö. Karaca, V. Scalcon, S.M. Meier-Menches, R. Bonsignore, J.M.J.L. Brouwer, F. Tonolo, A. Folda, M.P. Rigobello, F.E. Kühn, A. Casini, Characterization of Hydrophilic Gold(I) N-Heterocyclic Carbene (NHC) Complexes as Potent TrxR Inhibitors Using Biochemical and Mass Spectrometric Approaches, Inorg. Chem. 56 (2017) 14237-14250. doi:10.1021/acs.inorgchem.7b02345.

[22] N. Curado, N. Giménez, K. Miachin, M. Aliaga-Lavrijsen, M.A. Cornejo, A.A. Jarzecki, M. Contel, Preparation of Titanocene-Gold Compounds Based on Highly Active Gold(I)-N-Heterocyclic Carbene Anticancer Agents: Preliminary in vitro Studies in Renal and Prostate Cancer Cell Lines, ChemMedChem. 0 (2019). doi:10.1002/cmdc.201800796. 
Titanocene-Gold Complexes Containing N-Heterocyclic Carbene Ligands Inhibit Growth of Prostate, Renal, and Colon Cancers in Vitro, Organometallics. 35 (2016) 1218-1227. doi:10.1021/acs.organomet.6b00051.

[24] J.P. Owings, N.N. McNair, Y.F. Mui, T.N. Gustafsson, A. Holmgren, M. Contel, J.B. Goldberg, J.R. Mead, Auranofin and N-heterocyclic carbene gold-analogs are potent inhibitors of the bacteria Helicobacter pylori, FEMS Microbiol. Lett. 363 (2016). doi:10.1093/femsle/fnw148.

[25] A. Bindoli, M.P. Rigobello, G. Scutari, C. Gabbiani, A. Casini, L. Messori, Thioredoxin reductase: A target for gold compounds acting as potential anticancer drugs, Coord. Chem. Rev. 253 (2009) 1692-1707. doi:10.1016/j.ccr.2009.02.026.

[26] C. Gabbiani, G. Mastrobuoni, F. Sorrentino, B. Dani, M.P. Rigobello, A. Bindoli, M.A. Cinellu, G. Pieraccini,

L. Messori, A. Casini, Thioredoxin reductase, an emerging target for anticancer metallodrugs. Enzyme inhibition by cytotoxic gold(iii) compounds studied with combined mass spectrometry and biochemical assays, Med Chem Commun. 2 (2011) 50-54. doi:10.1039/C0MD00181C.

[27] S.M. Meier-Menches, C. Gerner, B.K. Keppler, M.A. Cinellu, A. Casini, Mass Spectrometry Uncovers Molecular Reactivities of Coordination and Organometallic Gold(III) Drug Candidates in Competitive Experiments That Correlate with Their Biological Effects, Inorg. Chem. 55 (2016) 4248-4259. doi:10.1021/acs.inorgchem.5b03000.

[28] B. Bertrand, A. Citta, I.L. Franken, M. Picquet, A. Folda, V. Scalcon, M.P. Rigobello, P. Le Gendre, A. Casini, E. Bodio, Gold(I) NHC-based homo- and heterobimetallic complexes: synthesis, characterization and evaluation as potential anticancer agents, J. Biol. Inorg. Chem. 20 (2015) 1005-1020. doi:10.1007/s00775-015-1283-1.

[29] A. Citta, E. Schuh, F. Mohr, A. Folda, M.L. Massimino, A. Bindoli, A. Casini, M.P. Rigobello, Fluorescent silver(I) and gold(I)-N-heterocyclic carbene complexes with cytotoxic properties: mechanistic insights, Met. Integr. Biometal Sci. 5 (2013) 1006-1015. doi:10.1039/c3mt20260g.

[30] E. Schuh, C. Pflüger, A. Citta, A. Folda, M.P. Rigobello, A. Bindoli, A. Casini, F. Mohr, Gold(I) carbene complexes causing thioredoxin 1 and thioredoxin 2 oxidation as potential anticancer agents, J. Med. Chem. 55 (2012) 5518-5528. doi:10.1021/jm300428v.

[31] H. Valdés, D. Canseco-González, J.M. Germán-Acacio, D. Morales-Morales, Xanthine based N-heterocyclic carbene (NHC) complexes, Spec. Issue Dedic. Prof Irina Beletskaya. $867 \quad(2018) \quad 51-54$. doi:10.1016/j.jorganchem.2018.01.008.

[32] O. Karaca, S.M. Meier-Menches, A. Casini, F.E. Kuhn, On the binding modes of metal NHC complexes with DNA secondary structures: implications for therapy and imaging, Chem. Commun. (2017). doi:0.1039/c7cc03074f. 

8637. doi:10.1093/nar/gkv862.

[34] A. Bernal, L. Tusell, Telomeres: Implications for Cancer Development, Int. J. Mol. Sci. 19 (2018) 294. doi:10.3390/ijms19010294.

[35] S. Neidle, Therapeutic Applications of Quadruplex Nucleic Acids, Academic Pr, London; Waltham, MA, 2011. 10.1016/C2009-0-30510-3.

[36] S. Neidle, Quadruplex nucleic acids as targets for anticancer therapeutics, Nat. Rev. Chem. 1 (2017) s41570017-0041-017. doi:10.1038/s41570-017-0041.

[37] B. Bertrand, L. Stefan, M. Pirrotta, D. Monchaud, E. Bodio, P. Richard, P. Le Gendre, E. Warmerdam, M.H. de Jager, G.M.M. Groothuis, M. Picquet, A. Casini, Caffeine-Based Gold(I) N-Heterocyclic Carbenes as Possible Anticancer Agents: Synthesis and Biological Properties, Inorg. Chem. 53 (2014) 2296-2303. doi:10.1021/ic403011h.

[38] N.W. Smith, A. Alonso, C.M. Brown, S.V. Dzyuba, Triazole-containing BODIPY dyes as novel fluorescent probes for soluble oligomers of amyloid Aß1-42 peptide, Biochem. Biophys. Res. Commun. 391 (2010) 1455-1458. doi:10.1016/j.bbrc.2009.12.091.

[39] CrysAlisPro Software System, Rigaku Oxford Diffraction, 2019.

[40] O.V. Dolomanov, L.J. Bourhis, R.J. Gildea, J.A.K. Howard, H. Puschmann, OLEX2: a complete structure solution, refinement and analysis program, J. Appl. Crystallogr. 42 (2009) 339-341. doi:10.1107/S0021889808042726. [41] G.M. Sheldrick, Crystal structure refinement with SHELXL, Acta Crystallogr. Sect. C Struct. Chem. 71 (2015) 3-8. doi:10.1107/S2053229614024218.

[42] G.M. Sheldrick, SHELXT - Integrated space-group and crystal-structure determination, Acta Crystallogr. Sect. A. 71 (2015) 3-8. doi:10.1107/S2053273314026370.

[43] J. Schindelin, I. Arganda-Carreras, E. Frise, V. Kaynig, M. Longair, T. Pietzsch, S. Preibisch, C. Rueden, S. Saalfeld, B. Schmid, J.-Y. Tinevez, D.J. White, V. Hartenstein, K. Eliceiri, P. Tomancak, A. Cardona, Fiji: an opensource platform for biological-image analysis, Nat. Methods. 9 (2012) 676.

[44] H. Schmidbaur, A. Schier, Aurophilic interactions as a subject of current research: an up-date, Chem. Soc. Rev. 41 (2012) 370-412. doi:10.1039/C1CS15182G.

[45] D. Renčiuk, J. Zhou, L. Beaurepaire, A. Guédin, A. Bourdoncle, J.-L. Mergny, A FRET-based screening assay for nucleic acid ligands, Quadruplex DNA Methods. 57 (2012) 122-128. doi:10.1016/j.ymeth.2012.03.020.

[46] J. Lennartsson, L. Rönnstrand, Stem Cell Factor Receptor/c-Kit: From Basic Science to Clinical Implications, Physiol. Rev. 92 (2012) 1619-1649. doi:10.1152/physrev.00046.2011. 
[47] S. Kyo, M. Takakura, T. Fujiwara, M. Inoue, Understanding and exploiting hTERT promoter regulation for diagnosis and treatment of human cancers, Cancer Sci. 99 (2008) 1528-1538. doi:10.1111/j.1349-7006.2008.00878.x.

[48] S. Masiero, R. Trotta, S. Pieraccini, S. De Tito, R. Perone, A. Randazzo, G.P. Spada, A non-empirical chromophoric interpretation of CD spectra of DNA G-quadruplex structures, Org. Biomol. Chem. 8 (2010) $2683-2692$. doi:10.1039/c003428b.

[49] N. Zhang, A.T. Phan, D.J. Patel, $(3+1)$ Assembly of three human telomeric repeats into an asymmetric dimeric G-quadruplex, J. Am. Chem. Soc. 127 (2005) 17277-17285. doi:10.1021/ja0543090.

[50] R. Bonsignore, A. Notaro, A.M.P. Salvo, A. Spinello, G. Fiasconaro, A. Terenzi, F. Giacalone, B.K. Keppler,

M. Giuliano, M. Gruttadauria, G. Barone, DNA-Binding and Anticancer Activity of Pyrene-Imidazolium Derivatives, ChemistrySelect. 1 (2016) 6755-6761. doi:10.1002/slct.201601502.

[51] R. Bonsignore, A. Terenzi, A. Spinello, A. Martorana, A. Lauria, A.M. Almerico, B.K. Keppler, G. Barone, Gquadruplex vs. duplex-DNA binding of nickel(II) and zinc(II) Schiff base complexes, J. Inorg. Biochem. 161 (2016) 115121. doi:10.1016/j.jinorgbio.2016.05.010.

[52] A. Terenzi, R. Bonsignore, A. Spinello, C. Gentile, A. Martorana, C. Ducani, B. Högberg, A.M. Almerico, A. Lauria, G. Barone, Selective G-quadruplex stabilizers: Schiff-base metal complexes with anticancer activity, RSC Adv. 4 (2014) 33245-33256. doi:10.1039/C4RA05355A.

[53] R. Bonsignore, F. Russo, A. Terenzi, A. Spinello, A. Lauria, G. Gennaro, A.M. Almerico, B.K. Keppler, G. Barone, The interaction of Schiff Base complexes of nickel(II) and zinc(II) with duplex and G-quadruplex DNA, J. Inorg. Biochem. 178 (2018) 106-114. doi:10.1016/j.jinorgbio.2017.10.010. 


\section{List of Figures, Schemes and Tables}

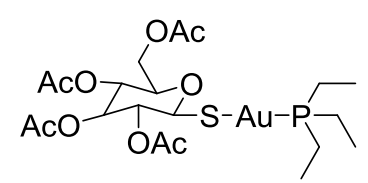

Auranofin

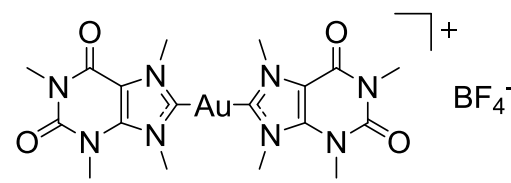

AuTMX

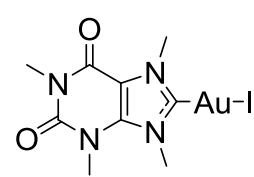

AuTMXI

Figure 1 - Chemical structures of gold(I) coordination and organometallic compounds as experimental anticancer agents.

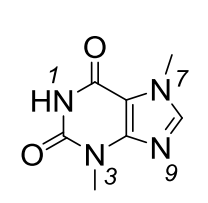

theobromine

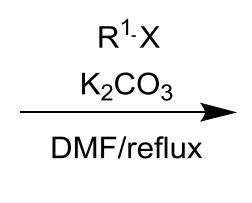

$\mathrm{R}^{1}=$

a)

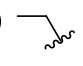

$39 \%$

c)

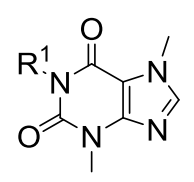

1a-d
$\frac{\begin{array}{c}\mathrm{Me}_{3} \mathrm{OBF}_{4} \\ \mathrm{Na}_{2} \mathrm{CO}_{3}\end{array}}{\mathrm{ACN} \text { (dry) }}$

2a-d b)

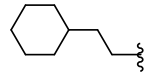

$20 \%$ d) $\mathrm{F}_{3} \mathrm{C}$ $66 \%$

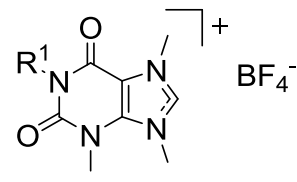

1. $\mathrm{Ag}_{2} \mathrm{O}, \mathrm{MeCN}$ (dry)

2. $\mathrm{Au}$ (tht) $\mathrm{Cl}, \mathrm{KI}$

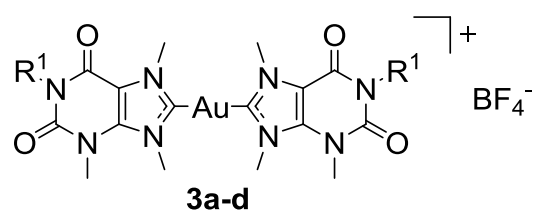

Scheme 1. Synthetic pathway leading to the Au(I) bis-NHC complexes 3a-d. 


$$
=R_{\text {AuTMXI }}^{2}
$$

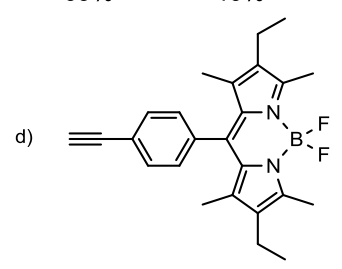

$68 \%$

Scheme 2. Synthetic pathway leading to complexes 4a-d.

A

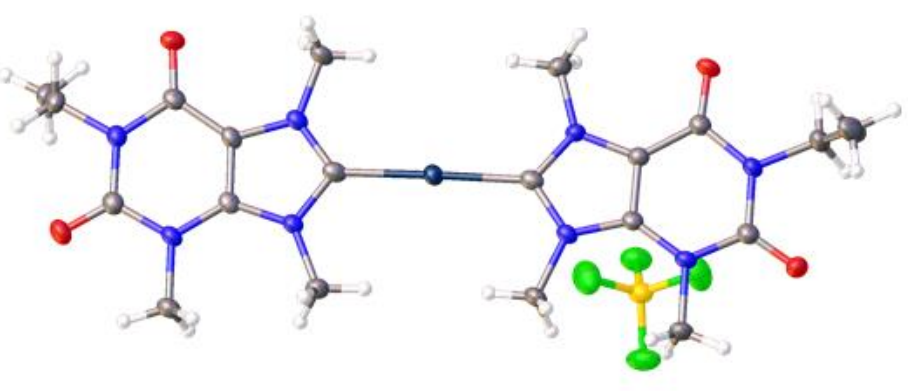

B

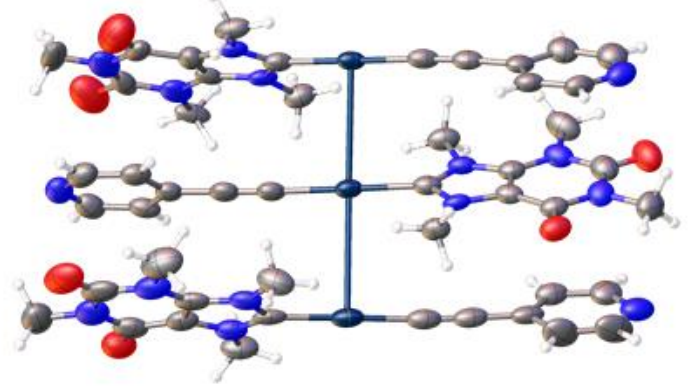

C

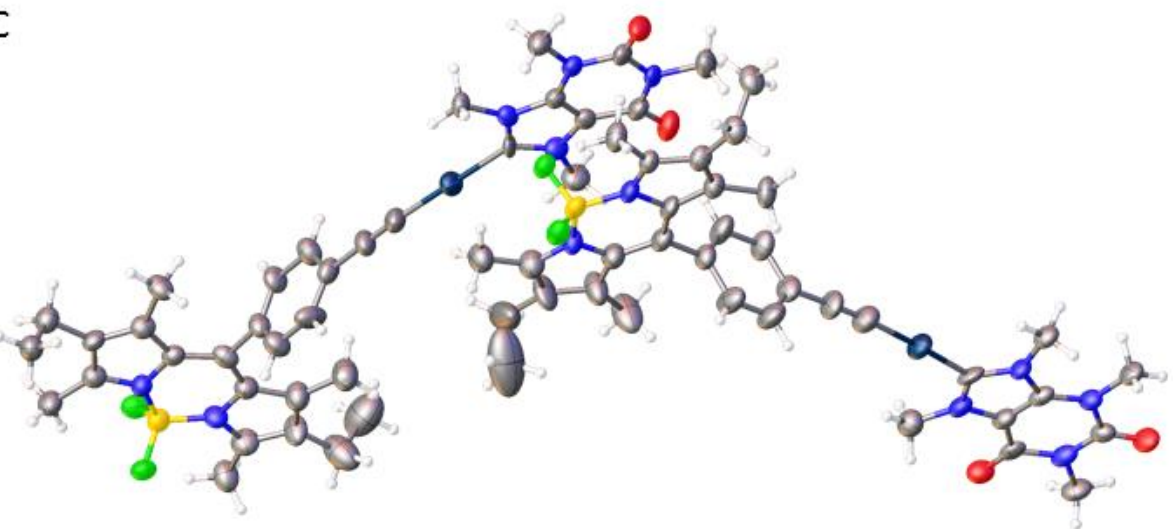

Figure 2. ORTEP plot of 3a (A), $\mathbf{4 b}(\mathrm{B})$ and $\mathbf{4 d}(\mathrm{C})$. Ellipsoids are shown at 50\% probability. 
Table 1 - Photophysical data for BODIPY-alkyne and 4d.

\begin{tabular}{lcccc}
\hline Compound & $\lambda \max (\mathbf{a b s})[\mathbf{n m}]$ & $\boldsymbol{\varepsilon} \max \left[\mathbf{L ~} \mathbf{~ m o l}^{-1} \mathbf{c m}^{-1}\right]$ & $\lambda_{\max (\mathrm{ems})}[\mathbf{n m}]$ & $\boldsymbol{\varphi}[\%]$ \\
\hline BODIPY-alkyne & 525 & 73 & 543 & $\mathbf{7 5}$ \\
\hline $\mathbf{4 d}$ & $295,377,526$ & 71 & 542 & $\mathbf{7 4}$
\end{tabular}

A

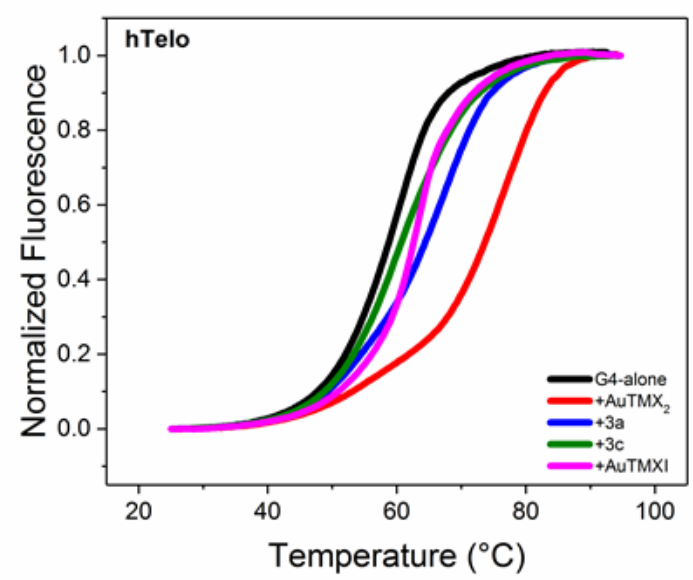

C

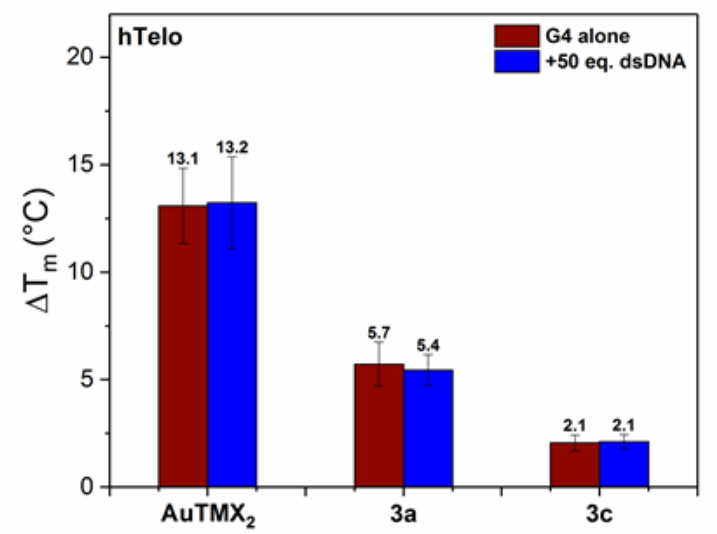

B
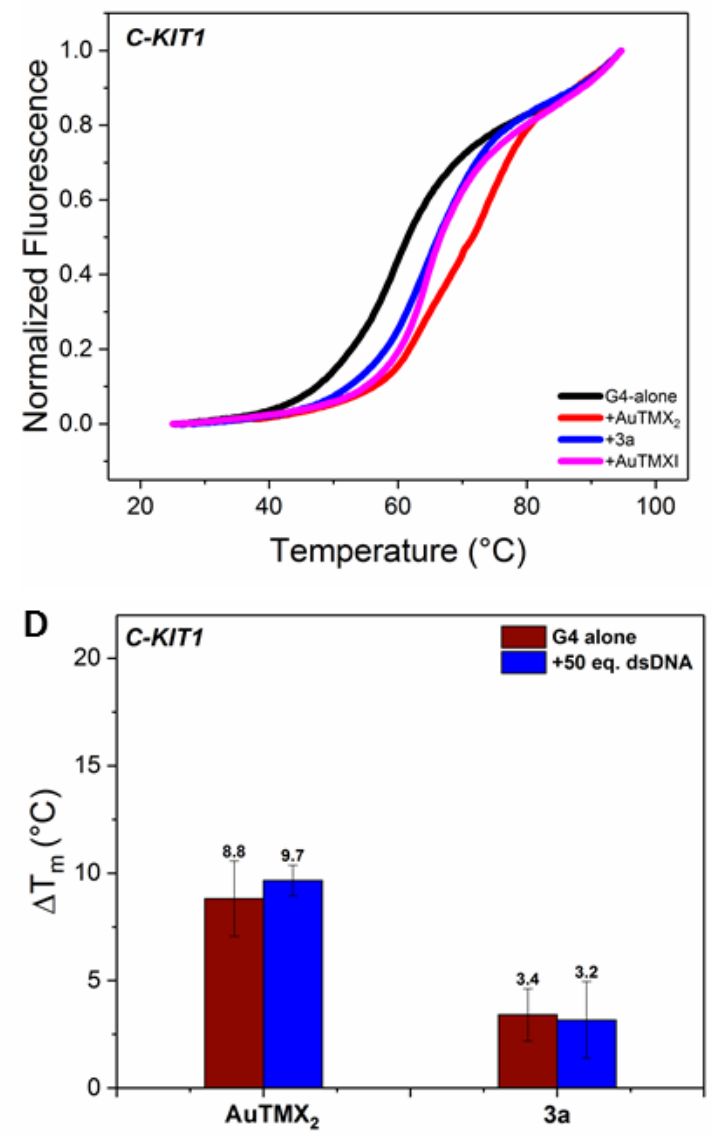

Figure 3. (A and B) Representative FRET DNA melting profiles of $0.2 \mu \mathrm{M}$ hTelo (A) and C-KITl (B) G4-DNA solutions in $54 \mathrm{mM}$ potassium cacodylate $(\mathrm{pH}=7.4)$ in presence of 5 equiv. of selected $\mathrm{Au}(\mathrm{I}) \mathrm{NHC}$ compounds; $(\mathbf{C}$ and $\mathbf{D}) \Delta \mathrm{T}_{\mathrm{m}}$ $\left({ }^{\circ} \mathrm{C}\right)$ of hTelo and $C$-KITI G4-DNA solutions in $54 \mathrm{mM}$ potassium cacodylate $(\mathrm{pH}=7.4)$ in presence of 5 equiv. of selected compound without (red bars) and with 50 equiv. of dsDNA (blue bars). Data are shown as mean \pm SEM of three independent experiments. 

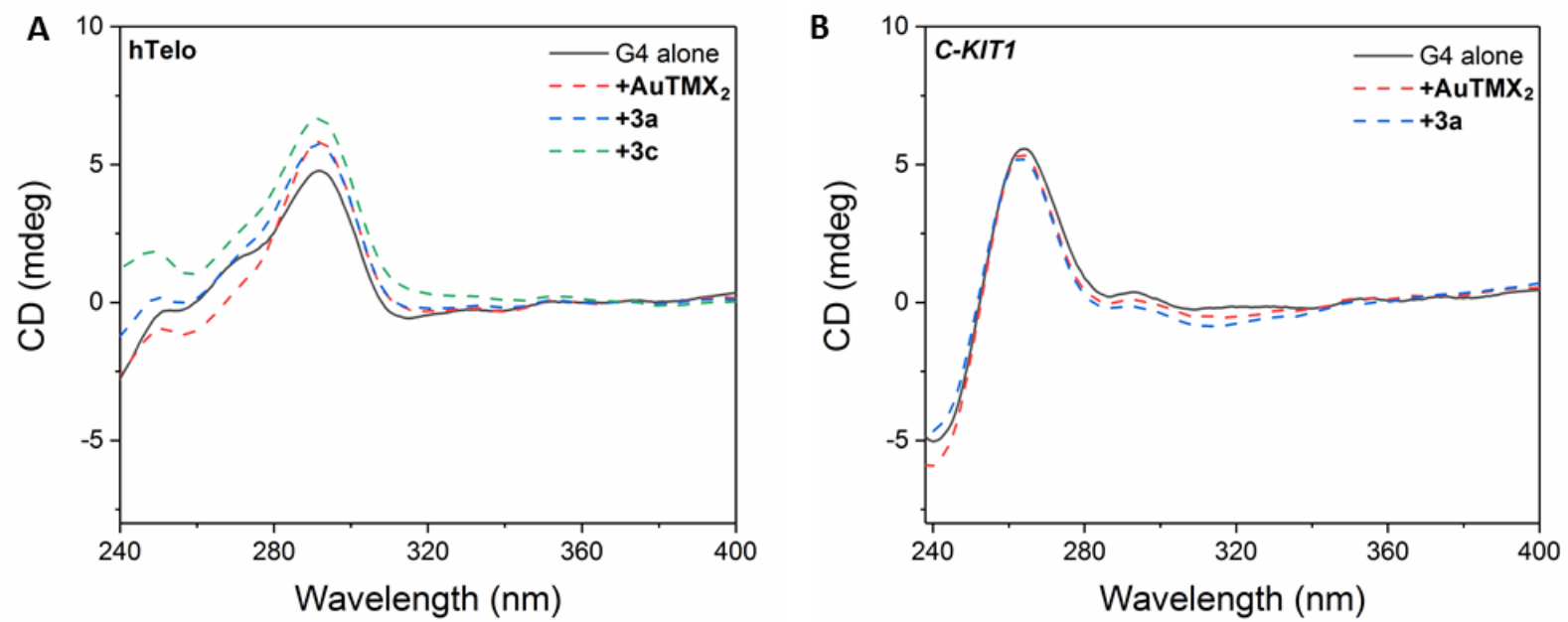

Figure 4. (A) CD spectra of $4 \mu \mathrm{M}$ hTelo (black solid line) and (B) C-KITI (black solid line) G4-DNA solutions in Tris$\mathrm{HCl} / \mathrm{KCl}(10 / 50 \mathrm{mM}, \mathrm{pH}=7.4)$ without or with of 5 equiv. of selected $\mathrm{Au}(\mathrm{I}) \mathrm{NHC}$ compounds (dashed lines). 
Table 2. Effective concentration of $50 \%$ growth inhibition $\left(\mathrm{EC}_{50}\right)$ for the $\mathrm{Au}(\mathrm{I}) \mathrm{NHC}$ complexes against different human cancer cell lines after $72 \mathrm{~h}$ incubation.

\begin{tabular}{|c|c|c|c|}
\hline & \multicolumn{3}{|c|}{$\mathbf{E C}_{50}(\mu \mathbf{M})^{\mathrm{a}}$} \\
\hline Compound & A375 & SKOV-3 & MCF-7 \\
\hline $\mathrm{AuTMX}_{2}$ & $>80$ & $>80$ & $>90$ \\
\hline AuTMXI & $>50$ & $37 \pm 10^{\mathrm{b}}$ & $>50$ \\
\hline $3 \mathbf{a}$ & $>90$ & $>100$ & $>80$ \\
\hline $3 b$ & $8.8 \pm 0.8$ & $13.0 \pm 0.9$ & $6.1 \pm 0.8$ \\
\hline $3 c$ & $28 \pm 3$ & $36 \pm 4$ & $18 \pm 2$ \\
\hline $4 a$ & $>100$ & $>100$ & $>50$ \\
\hline $4 b$ & $>100$ & $>100$ & $36(n=2)$ \\
\hline $4 c$ & $>80$ & $>50$ & $16 \pm 5$ \\
\hline $4 d^{c}$ & $>80$ & $>100$ & $>100$ \\
\hline
\end{tabular}

${ }^{\text {a }}$ Data are presented as mean \pm standard deviation of at least three independent experiments, unless otherwise specified. $\mathrm{n}=$ number of experiments.

${ }^{\text {b}}$ Value taken from ref. 34.

${ }^{\mathrm{c}} \mathrm{EC}_{50}$ values determined with CellTiterBlue ${ }^{\circledR}$ assay. 


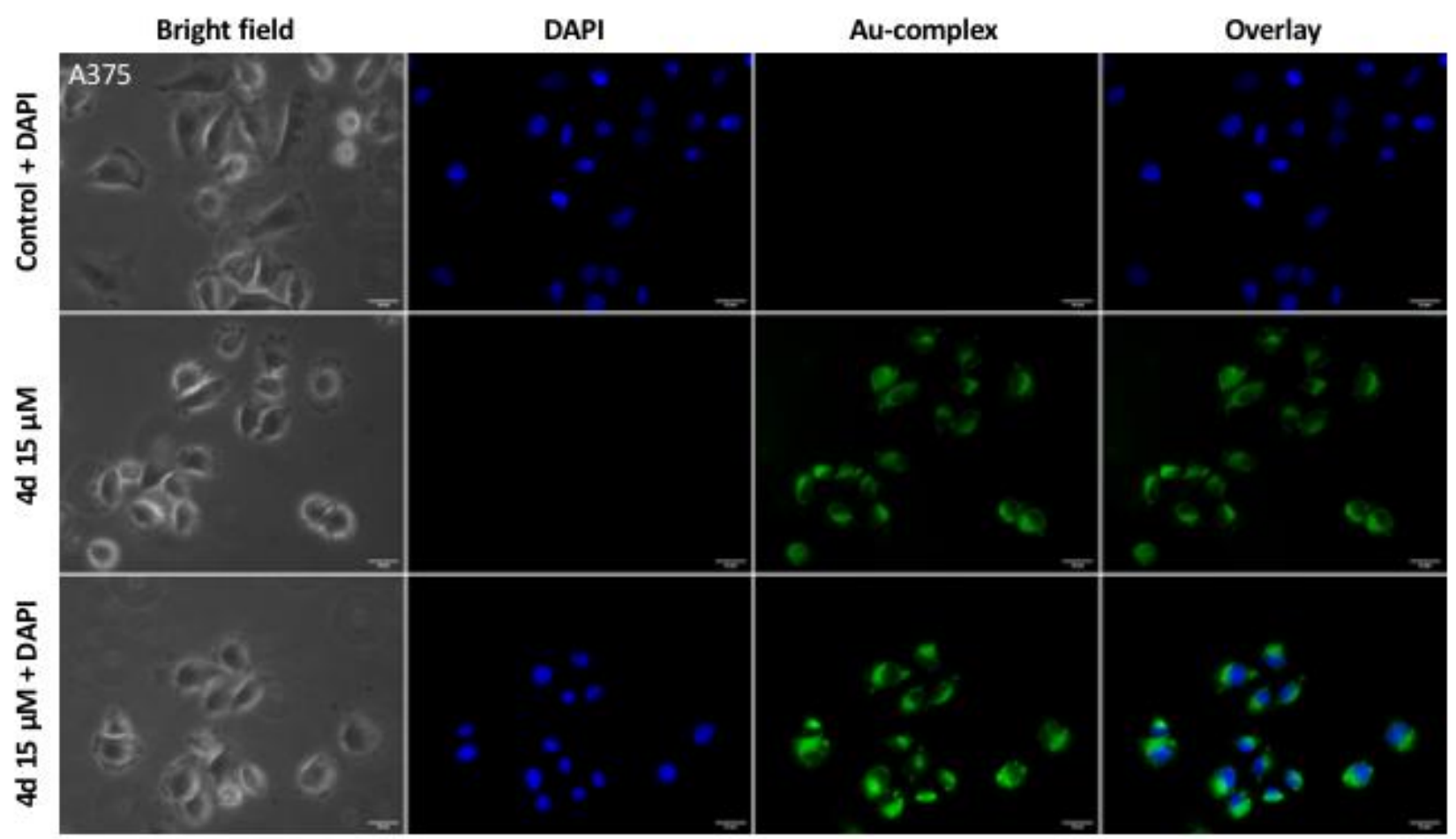

Figure 5. Bright field and fluorescence microscopy images of fixed human A375 melanoma cells either as untreated controls (top row) or treated with $\mathbf{4 d}$ for $2 \mathrm{~h}$ (middle and bottom row). DAPI = 4',6-diamidino-2-phenylindole. Scale bar represents $20 \mu \mathrm{m}$. 